\title{
Tropoelastin Implants That Accelerate Wound Repair
}

DOI:

10.1002/adhm.201701206

\section{Document Version}

Accepted author manuscript

Link to publication record in Manchester Research Explorer

\section{Citation for published version (APA):}

Mithieux, S. M., Aghaei-Ghareh-Bolagh, B., Yan, L., Kuppan, K. V., Wang, Y., Garces-Suarez, F., Li, Z., Maitz, P. K., Carter, E. A., Limantoro, C., Chrzanowski, W., Cookson, D., Riboldi-Tunnicliffe, A., Baldock, C., Ohgo, K., Kumashiro, K. K., Edwards, G., \& Weiss, A. S. (2018). Tropoelastin Implants That Accelerate Wound Repair. Advanced Healthcare Materials. https://doi.org/10.1002/adhm.201701206

\section{Published in:}

Advanced Healthcare Materials

\section{Citing this paper}

Please note that where the full-text provided on Manchester Research Explorer is the Author Accepted Manuscript or Proof version this may differ from the final Published version. If citing, it is advised that you check and use the publisher's definitive version.

\section{General rights}

Copyright and moral rights for the publications made accessible in the Research Explorer are retained by the authors and/or other copyright owners and it is a condition of accessing publications that users recognise and abide by the legal requirements associated with these rights.

\section{Takedown policy}

If you believe that this document breaches copyright please refer to the University of Manchester's Takedown Procedures [http://man.ac.uk/04Y6Bo] or contact uml.scholarlycommunications@manchester.ac.uk providing relevant details, so we can investigate your claim.

\section{OPEN ACCESS}




\section{Advanced Healthcare Materials Tropoelastin implants that accelerate wound repair \\ --Manuscript Draft--}

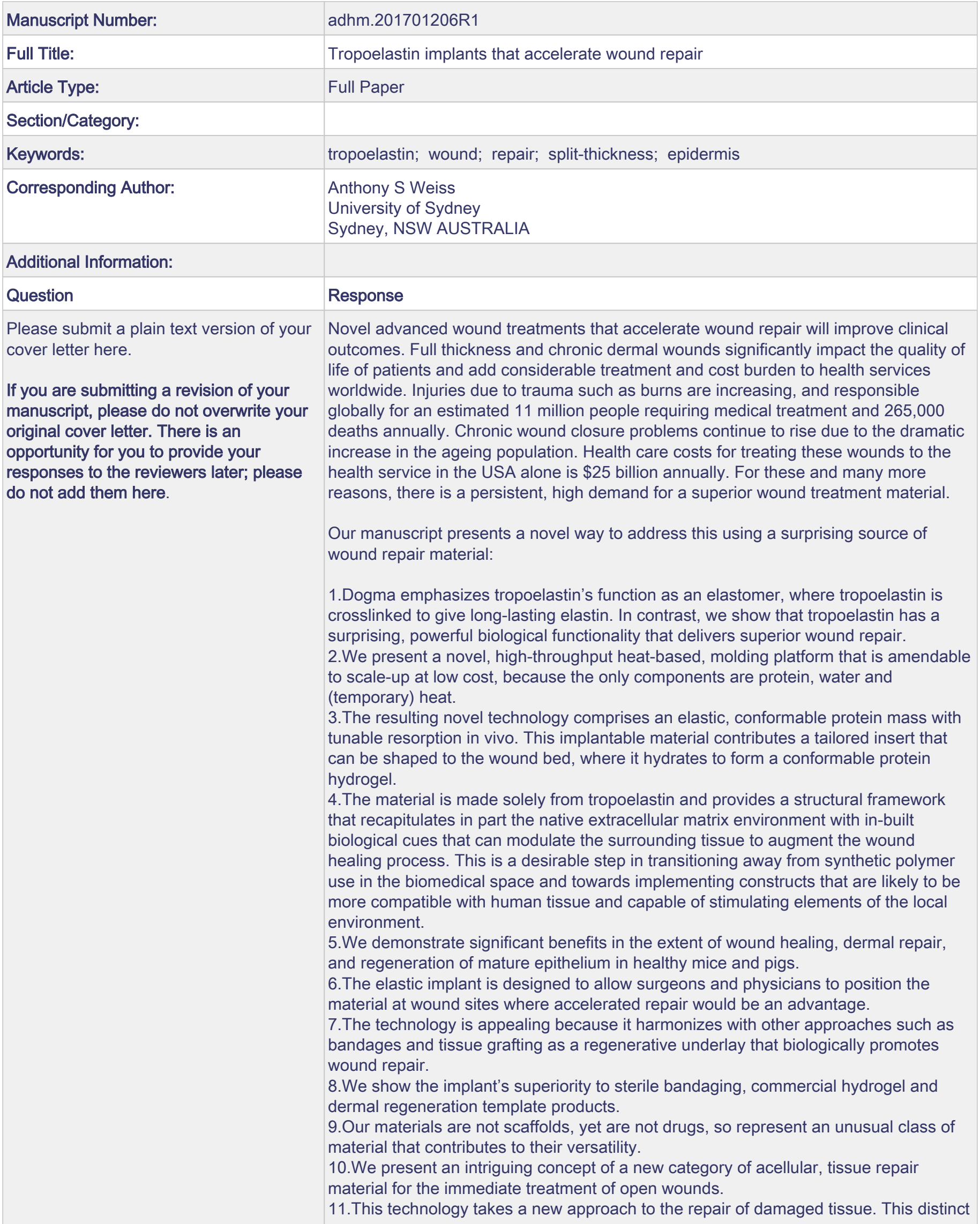




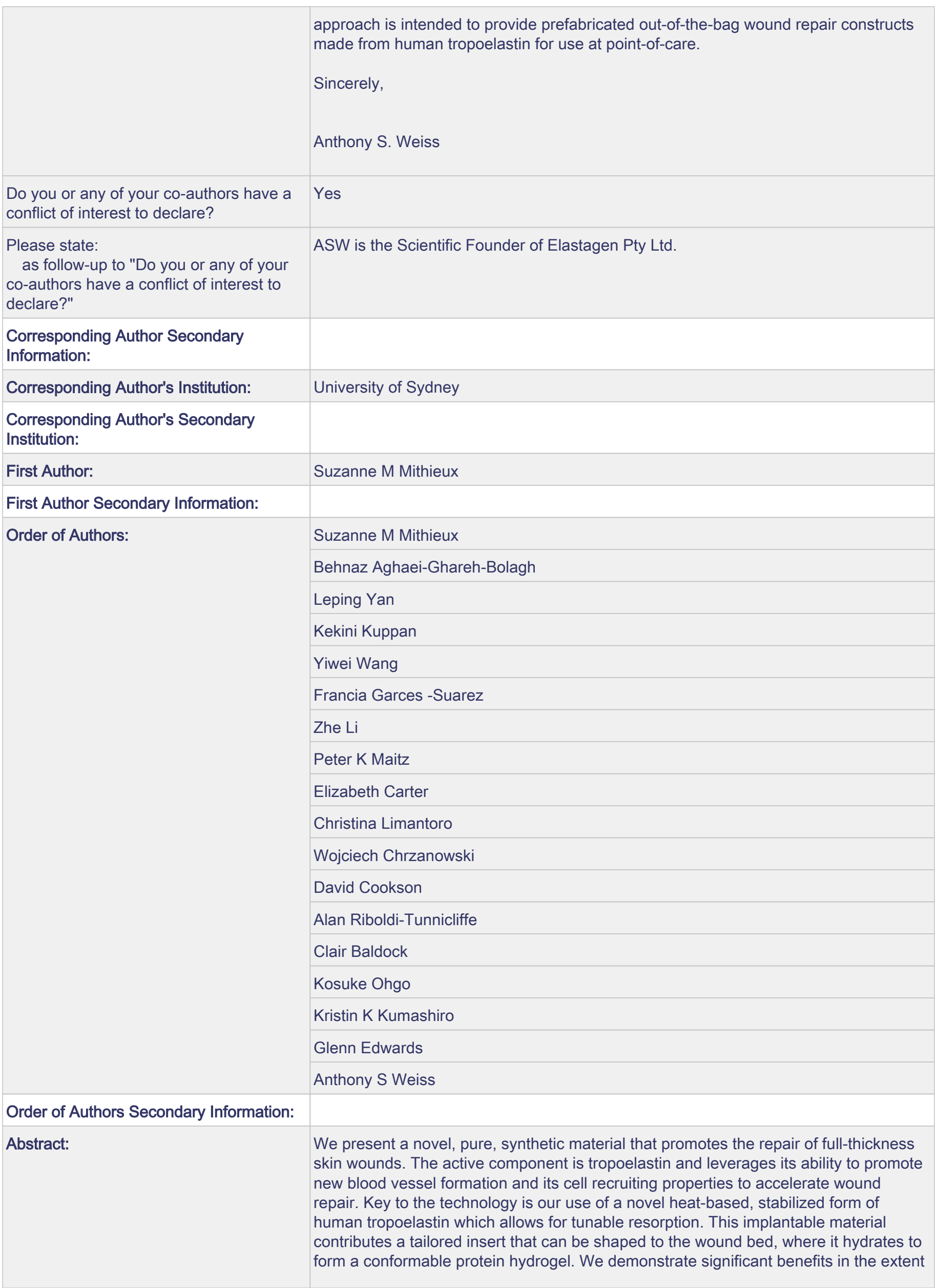


of wound healing, dermal repair, and regeneration of mature epithelium in healthy pigs. The implant is compatible with initial co-treatment with full and split-thickness skin grafts. We show the implant's superiority to sterile bandaging, commercial hydrogel and dermal regeneration template products. On this basis, we provide a new concept for a prefabricated tissue repair material for point-of-care treatment of open wounds. 


\section{WILEY-VCH}

DOI: $10.1002 /$ ((please add manuscript number))

Article type: Full Paper

Title: Tropoelastin implants that accelerate wound repair

Suzanne M. Mithieux, Behnaz Aghaei-Ghareh-Bolagh, Leping Yan, Kekini V. Kuppan, Yiwei Wang, Francia Garces-Suarez, Zhe Li, Peter K. Maitz, Elizabeth Carter, Christina Limantoro, Wojciech Chrzanowski, David Cookson, Alan Riboldi-Tunnicliffe, Clair Baldock, Kosuke Ohgo, Kristin K. Kumashiro, Glenn Edwards and Anthony S. Weiss*

Dr. S. M. Mithieux, B. Aghaei-Ghareh-Bolagh, K.V. Kuppan, Dr. L. Yan, Prof. A.S. Weiss School of Life and Environmental Sciences, University of Sydney, NSW 2006, Australia Charles Perkins Centre, University of Sydney, NSW 2006, Australia

Email: tony.weiss@sydney.edu.au

K.V. Kuppan

Heart Research Institute, University of Sydney, NSW 2006, Australia

Dr. Y. Wang, F. Garces-Suarez, Dr. Z. Li, Prof. P. K. Maitz

Burns Research Group, ANZAC Research Institute, University of Sydney, Concord, NSW 2139, Australia

Dr. E. Carter

Vibrational Spectroscopy Core Facility and Faculty of Chemistry, University of Sydney, NSW 2006, Australia

C. Limantoro, Dr. W. Chrzanowski

Faculty of Pharmacy, University of Sydney, NSW 2006, Australia

Australian Institute for Nanoscale Science and Technology, University of Sydney, NSW 2006, Australia

Dr. D. Cookson, Dr. A. Riboldi-Tunnicliffe

Australian Synchrotron ANSTO, VIC 3168, Australia

Prof. C. Baldock

Wellcome Trust Centre for Cell-Matrix Research, Division of Cell Matrix Biology and Regenerative Medicine, School of Biological Sciences, Manchester Academic Health Centre, University of Manchester, Manchester, UK

Dr. K. Ohgo, Prof. K. K. Kumashiro

Department of Chemistry, University of Hawaii, Honolulu, HI 96822, USA

Prof. Glenn Edwards

School of Animal and Veterinary Sciences, Charles Sturt University, NSW 2678, Australia

Prof. A.S. Weiss

Bosch Institute, University of Sydney, NSW 2006, Australia

Keywords: tropoelastin, wound, repair, split-thickness, epidermis 


\title{
WILEY-VCH
}

\begin{abstract}
We present a novel, pure, synthetic material that promotes the repair of full-thickness skin wounds. The active component is tropoelastin and leverages its ability to promote new blood vessel formation and its cell recruiting properties to accelerate wound repair. Key to the technology is our use of a novel heat-based, stabilized form of human tropoelastin which allows for tunable resorption. This implantable material contributes a tailored insert that can be shaped to the wound bed, where it hydrates to form a conformable protein hydrogel. We demonstrate significant benefits in the extent of wound healing, dermal repair, and regeneration of mature epithelium in healthy pigs. The implant is compatible with initial cotreatment with full and split-thickness skin grafts. We show the implant's superiority to sterile bandaging, commercial hydrogel and dermal regeneration template products. On this basis, we provide a new concept for a prefabricated tissue repair material for point-of-care treatment of open wounds.
\end{abstract}

\section{Introduction}

Full thickness and chronic dermal wounds significantly impact the quality of life of patients and add considerable treatment and cost burden to health services worldwide. Injuries due to trauma such as burns are increasing, and responsible globally for an estimated 11 million people requiring medical treatment and 265,000 deaths annually. ${ }^{[1]}$ Chronic wound closure problems continue to rise due to the dramatic increase in the ageing population. ${ }^{[2]}$ Health care costs for treating these wounds to the health service in the USA alone is $\$ 25$ billion annually. ${ }^{[3]}$ Current standards of care includes debridement of the wound followed by treatment with a hydrogel to manage the wound environment, along with an absorbent dressing and, where required, negative pressure and antibiotic therapy. ${ }^{[4]}$ Novel advanced wound treatments that accelerate wound repair are vitally required to improve clinical outcomes. 


\section{WILEY-VCH}

Wound repair is one of the most complex biological processes exhibited by human tissue. ${ }^{[5]}$ It progresses through four overlapping phases - hemostasis, inflammation, proliferation and maturation. ${ }^{[6]}$ Following injury, blood flow in the wound is reduced through vasoconstriction and clot formation. The blood clot is comprised of cytokines and growth factors which facilitate the activation of the inflammatory process. The inflammation phase is marked by recruitment of circulating inflammatory cells including neutrophils, monocytes and macrophages to the wound site. During the proliferation stage, dermal and epidermal integrity is restored. Fibroblasts migrate to the wound site where they deposit extracellular matrix (ECM) in the form of granulation tissue. Transport of oxygen, nutrients, cells and growth factors is augmented through increased vascularization and the barrier function of the skin is re-established through formation of a stratified keratinized epithelium. In the maturation phase collagen organization takes place, fibroblasts undergo apoptosis and the wound becomes less vascularized.

Current approaches to wound treatment rely on peripheral tissue's ability to construct the essential structural ECM components rather than enhancement of the endogenous wound repair phases. Next generation wound treatments that aim to facilitate the natural wound healing processes have been called for. Particularly sought-after approaches include those that target modulation of immune cells, enhancement of fibroblast and keratinocyte invasion and migration, and promotion of angiogenesis. ${ }^{[7]} \mathrm{We}$ are accumulating evidence that the ECM protein tropoelastin participates in many of these processes. Tropoelastin triggers rapid dermal fibroblast up-regulation of chemokines that participate in pro-inflammatory cellsignaling, including the recruitment of neutrophils into a wound site ${ }^{[8]}$ Tropoelastin and tropoelastin-based materials support attachment, spreading, infiltration and proliferation of dermal fibroblasts. ${ }^{[9]}$ Tropoelastin supports endothelial progenitor cell binding via an integrin 


\section{WILEY-VCH}

$\alpha \mathrm{V} \beta 3$ mechanism ${ }^{[10]}$ and promotes eNOS formation, while this same integrin plays a key role in angiogenesis. ${ }^{[1]}$ Tropoelastin promotes endothelial cell growth ${ }^{[12]}$ and signaling ${ }^{[13]}$ and coordinately accelerates the formation of perfused blood vessel de novo from organized endothelial cells due to the enhanced release of a plethora of angiogenic cytokines to nurture local tissue growth. ${ }^{[14]}$ While these features suggest that tropoelastin has the potential to promote wound repair, this ability has not been demonstrated.

We show here, for the first time, that tropoelastin promotes full-thickness dermal wound repair. To enable the placement of tropoelastin, we introduce a novel, high-throughput heatbased, molding procedure. Unlike previous tropoelastin-based biomaterials that require chemical modification ${ }^{[15]}$ to facilitate stabilization, the ensuing material is made solely from tropoelastin. The process delivers a structural framework that recapitulates in part the native ECM environment with in-built biological cues that can modulate the surrounding tissue to augment the wound healing process. This is a desirable step in transitioning away from synthetic polymer use in the biomedical space and towards implementing constructs that are likely to be more compatible with human tissue and capable of stimulating elements of the local environment. ${ }^{[16]}$ By adjusting the heating time, we obtain a commensurate change in the resorption time of these materials. This implantable technology provides tropoelastin in the form of a material that can be shaped to the wound bed. We show here that the elastic implant promotes wound repair in pigs. The technology is appealing because it harmonizes with other approaches such as bandages and tissue grafting as a regenerative underlay that biologically promotes wound repair. The elastic implant is designed to allow surgeons and physicians to position the material at wound sites where accelerated repair would be an advantage. This technology takes a new approach to the repair of damaged tissue. This distinct approach is intended to provide prefabricated wound repair constructs made from human tropoelastin for point-of-care application. 


\section{WILEY-VCH}

\section{Results and Discussion}

\subsection{Material fabrication and characterization}

In order to introduce tropoelastin into the wound bed, we designed a novel process that uses tropoelastin as the sole component to generate shaped protein constructs. This versatile, straightforward thermosetting process comprises three main stages: (1) tropoelastin is dissolved in a solvent and cast into or onto a mold; (2) the solution is dried in its cast form, and then (3) heated to $160^{\circ} \mathrm{C}$ for a defined period of time. Typical production is described in Figure 1. We have tested this process with tubes, sponges and micropatterned films that were formed using diverse combinations of casting and drying techniques including electrospinning, mold casting, lyophilization and air-drying. We have named the resulting material HeaTro to reflect its simple combination of heat and tropoelastin.

HeaTro becomes increasingly stable with extended heating times. We investigated the source of this thermal stabilization. SDS-PAGE analysis under reducing conditions, of protein leached from HeaTro samples after soaking in water for $16 \mathrm{~h}$ indicated the presence of both monomer, dimer, trimer and higher multimer tropoelastin species (Figure 2a). With increasing heat treatment times the amount of leachable monomer decreased until no monomer could be detected following a $16 \mathrm{~h}$ heat treatment. The extent of tropoelastin multimerization increased with increasing heat treatment times such that protein arrays too large to enter the gel were evident in the gel wells following 4 and $8 \mathrm{~h}$ heat treatments and no detectable multimer leachables were present following a $16 \mathrm{~h}$ heat treatment. The presence of multimers suggests the formation of heat-induced cross-links, which increase in number with prolonged heating. However, we cannot exclude an alternative model of tropoelastin stabilization due to excluded water between closely-spaced tropoelastin molecules. Thermal stabilization of gelatin, ${ }^{[17]}$ collagen, ${ }^{[18]}$ keratin $^{[19]}$ and silk ${ }^{[20]}$ has generally been attributed to 


\section{WILEY-VCH}

cross-link formation. For zero-length cross-link formation to occur tropoelastin molecules must be in close contact. AFM analysis (Figure $\mathbf{2 b}$ ) of surface topography indicated HeaTro (8 $\mathrm{h}$ heat treatment) comprises tightly packed molecules with minimal surface features at $<$ $2.5 \mathrm{~nm}$ in height and no obvious spacing between molecules. The swelling behavior of the HeaTro constructs in water and PBS (Figure 2c and 2d) also pointed to cross-link formation. Swelling behavior reflects the extent of cross-linking, where the degree of swelling is limited with increasing cross-link numbers. ${ }^{[21]}$ At $25^{\circ} \mathrm{C}$, HeaTro constructs stabilized for $2 \mathrm{~h}$ and $4 \mathrm{~h}$ at $160^{\circ} \mathrm{C}$ swelled so much in water that accurate measurements could not be made. Samples heated for $8 \mathrm{~h}$ at $160^{\circ} \mathrm{C}$ absorbed $180 \pm 6 \mathrm{~g}$ water/g protein compared to $74 \pm 6 \mathrm{~g}$ water/g protein for HeaTro constructs stabilized for $16 \mathrm{~h}$. At $25^{\circ} \mathrm{C}$, HeaTro constructs stabilized for 4,8 or 16 $\mathrm{h}$ at $160^{\circ} \mathrm{C}$ absorbed $5.8 \pm 0.3,4.8 \pm 0.2$ and $2.8 \pm 0.01 \mathrm{~g} \mathrm{PBS} / \mathrm{g}$ protein respectively. This behavior reflects the swelling properties of chemically and enzymatically cross-linked tropoelastin constructs. For example, at $20^{\circ} \mathrm{C}$ tropoelastin chemically cross-linked with bis(sulfosuccinimidyl) suberate absorbs $48 \pm 0.6 \mathrm{~g}$ water/g protein and $4.0 \pm 0.4 \mathrm{~g}$ PBS/g protein, while at $25^{\circ} \mathrm{C}$ tropoelastin enzymatically cross-linked through the actions of a Pichia pastoris lysyl oxidase absorbs $73 \mathrm{~g}$ water/g protein and $5.4 \mathrm{~g}$ PBS/g protein. ${ }^{[15 \mathrm{c}, 22]}$ Reduced cross-link numbers are likely to account for the increase in water absorption by HeaTro compared to chemically cross-linked tropoelastin. Increasing heating times at $160^{\circ} \mathrm{C}$ led to decreasing water and PBS absorption indicating that heating time could be used as a means to tune the swelling ratio of HeaTro.

The amino acid content of tropoelastin and HeaTro was indistinguishable (data not shown). This pointed to either no or hydrolyzable crosslinks in HeaTro. Examples of hydrolyzable cross-links typical of thermally stabilized proteins include amide and ester bonds formed between the negatively charged side chain carboxyl groups of glutamic and aspartic acid residues and either amine-containing or hydroxyl-containing amino acid side chains 


\section{WILEY-VCH}

respectively. ${ }^{[20 b]}$ In particular the number of isopeptide cross-links formed between the $\varepsilon^{-}$ amino group of lysine residues and the side-chain carboxyl groups of negatively charged residues have been shown to increase with the extent of heat treatment. ${ }^{[23]}$ While there are ample amine- and hydroxyl-containing sidechains in each tropoelastin molecule, such stabilization had not been expected to work because this molecule has an insufficiency of one aspartic and two glutamic acid residues that combined comprise less than $0.5 \%$ of the molecule. ${ }^{[24]}$ Peak intensities of peptide profiles from Lys-C digested monomer tropoelastin and tropoelastin dimer leached from a HeaTro sample thermally stabilized for $2 \mathrm{~h}$ were compared (Table S1). We consistently found that peak intensities of peptides encompassing the aspartic acid residue at position 72 in domain 6 of tropoelastin were at least two-fold higher in the monomer compared to the dimer. This was also the case for a peptide encompassing the C-terminal domains 31-36. No other peptides displayed such a change between monomer and dimer. The decrease in peak intensities in the dimer implicate these peptides in a possible crosslink. The proximity of domain 6 and the C-terminus in adjacent molecules is seen in lysyl oxidase ${ }^{[22]}$ and chemical ${ }^{[25]}$ crosslinking studies and is consistent with the endogenous head-to-tail assembly model. ${ }^{[26]}$

HeaTro constructs are inelastic when dry and elastic when wet. Mechanical studies demonstrated the elastic nature of the material; unstretched and stretched HeaTro sponges are shown in Figure 2e. Stress/strain curve analysis of the material (Figure 2f) gave an average Young's Modulus of $18 \pm 1 \mathrm{kPa}(\mathrm{n}=4)$ and an average elongation before break of $160 \pm 50 \%$. These values contrast with naturally cross-linked elastin which has a Young's Modulus of 300 - $600 \mathrm{kPa} \cdot{ }^{[27]}$ Tropoelastin monomer, and tropoelastin enzymatically crosslinked with a lysyl oxidase from Pichia pastoris, or chemically cross-linked with glutaraldehyde, have reported Young's moduli of $3 \mathrm{kPa},{ }^{[26]} 8-12 \mathrm{kPa}^{[22]}$ and $33 \pm 5 \mathrm{kPa}^{[15 \mathrm{~d}]}$ respectively. This is consistent with a model where heat stabilization generates a similar level of cross-linking effect on 


\section{WILEY-VCH}

monomer tropoelastin as these in vitro polymerizing methods.

Vibrational spectroscopy of HeaTro indicated that secondary structure changes are unlikely to contribute significantly to the thermal stabilization of tropoelastin because there were no detected changes in the position, line-shape or intensity of the conformationally-sensitive Amide bands. Furthermore, the secondary structure band positions for HeaTro are similar to those reported for human elastin. FTIR analysis (Figure 3a) displayed Amide I, II and III that were positioned at $1655,1545,1237 \mathrm{~cm}^{-1}$ respectively for tropoelastin which were indistinguishable from 1654, 1545 and $1238 \mathrm{~cm}^{-1}$ respectively for HeaTro. The positions of the Amide bands are similar to those reported for human elastin which are observed at 1660, 1540 and $1238 \mathrm{~cm}^{-1}$ respectively. ${ }^{[28]}$ Second derivative analysis of tropoelastin and HeaTro confirmed no significant spectral differences existed between the samples, with contributions from $\alpha$-helix $\left(1653 \mathrm{~cm}^{-1}\right)$ and $\beta$-structure $\left(1632 \mathrm{~cm}^{-1}\right)$. The similarity of the molecular structure of tropoelastin and HeaTro was further confirmed by Raman spectroscopy (Figure 3b) with the Amide I band centered at $1659 \mathrm{~cm}^{-1}$ for both tropoelastin and HeaTro. These values are similar to human elastin, where Raman Amide I and III peaks were reported at 1669 and $1246 \mathrm{~cm}^{-1} \cdot{ }^{[28]}$ Second derivative analysis of the Raman spectra revealed no significant spectral differences exist between the two samples. The Amide III band supported contributions from peaks at $1272 \mathrm{~cm}^{-1}\left(\alpha\right.$-helix) and $1250 \mathrm{~cm}^{-1}(\beta$-structure/random coil) for both samples.

The similarity of tropoelastin and HeaTro was also verified by Solid-state Cross-Polarization Magic Angle Spinning Carbon-13 NMR analysis (Figure 3c) which presented comparable spectra regardless of heat treatment.

Small and wide angle $\mathrm{x}$-ray scattering characterization verified that tropoelastin and HeaTro 


\section{WILEY-VCH}

samples were indistinguishable at lower scattering angles $\left(q<0.1 \AA^{-1}\right)($ Figure 3d). At higher scattering angles $(q>0.5 \AA-1)$ they were also comparable. On deconvolution of the diffraction pattern, tropoelastin gave rise to Gaussian fitted peaks at 0.74 and $1.45 \AA^{-1}$ that corresponded to real space inter- or intra-molecular distances of approximately 8.5 and $4.3 \AA$. HeaTro displayed similar peaks at 0.73 and $1.41 \AA^{-1}$ corresponding to distances of $\sim 8.6$ and $4.5 \AA$ respectively. These results approach the wide angle diffraction data of relaxed equine nuchal elastin with diffraction peaks at approximately 9.3 and $4.5 \AA{ }^{[29]}$ these values have been used to attribute molecular conformation and organization within elastin fibers to intraand inter-molecular hydrophobic interactions. ${ }^{[30]}$

Collectively, these amino acid analysis, vibrational spectroscopy, NMR, and small and wide angle x-ray scattering data demonstrate that the heating process that converts tropoelastin to HeaTro does not detectably alter the primary or secondary structure of the molecule. We conclude that tropoelastin treated in this way is minimally affected by heat.

\subsection{In vitro performance of HeaTro constructs}

We quantified cellular responses in order to assess whether HeaTro retained the biological efficacy of tropoelastin-based constructs, including the ability to promote fibroblast growth. In a manner typical of tropoelastin-based and tropoelastin-coated materials, ${ }^{[31]}$ HeaTro supported the attachment, spreading and proliferation of human dermal fibroblasts (HDF). The proliferation of these cells cultured on HeaTro over a 7-day period was demonstrated through a significant increase in DNA content (Figure 4a) and Hoechst-stained nuclei (Figure 4b). To further explore the biological efficacy of the material, we assessed whether solubilized HeaTro retained tropoelastin's known ability to promote cell migration. ${ }^{[32]}$ In an in vitro wound healing model ${ }^{[33]}$ HDFs cultured in media containing solubilized HeaTro displayed a significant increase in their rate of movement $(0.013 \pm 0.001 \mu \mathrm{m} / \mathrm{s})$ across a 


\section{WILEY-VCH}

scratch introduced through a confluent layer of cells compared to cells in control medium $(0.01 \pm 0.0007 \mu \mathrm{m} / \mathrm{s})($ Figure $4 \mathbf{c})$.

To model the effect of exposure to serum in a biological environment we placed HeaTro constructs in $100 \% \mathrm{FBS}$ at $37^{\circ} \mathrm{C}$ for 7 days. A heat treatment time of $2 \mathrm{~h}$ resulted in the release of $\sim 80 \%$ of the original mass of HeaTro samples after 7 days. In contrast, stabilization at $160^{\circ} \mathrm{C}$ for 8 or $10 \mathrm{~h}$ resulted in $\sim 50 \%$ and $37 \%$ respectively of the material being released under the same conditions (Figure 4d). These results demonstrate how HeaTro constructs can be tailored for either immediate solubilization or longer persistence in situ by simply adjusting their heat stabilization times.

\subsection{In vivo performance of HeaTro constructs}

A mouse full-thickness wound model with autografting was used to assess in vivo persistence. H\&E staining 2 weeks after subcutaneous implantation in mice showed tolerance with no evidence of adverse effects (data not shown). VVG-stained sections verified the stabilizing effect of longer heating times on HeaTro: two weeks after implantation HeaTro-2, -4, -6 and 8 samples, which were heat-stabilized for $2,4,6$ and $8 \mathrm{hr}$ respectively, were detected in 0,17 , 33 or $67 \%$ of the tissue explants respectively (n=4-6) (Figure 4e and 4f). In addition, perfused blood vessels were obvious within the vicinity of the HeaTro implants, in contrast to areas surrounding the Integra Dermal Regeneration Template (IDRT) implants or skin tissue from outside the wound. Acceleration of early stage vascularization can facilitate progression through the wound repair process. ${ }^{[14 a]}$

In contrast to mouse skin which heals by contraction, pig skin heals by a process that more closely resembles that of human subjects. ${ }^{[34]}$ A pig full thickness wound model was used to examine the utility of HeaTro in dermal wound healing. HeaTro-2 and -8 constructs, designed 


\section{WILEY-VCH}

to bracket the range that had been explored in mice, provided either a short or an extended treatment respectively. These were evaluated with and without split thickness grafting. Four control treatments were used: open wound, split thickness graft, IDRT without the silicone layer, and Solosite as a representative carboxymethylcellulose-based hydrogel treatment commonly used for the management of surgical incisions and ulcers. Figure 5 shows typical wound sites of non-grafted and grafted samples at both the time of implantation and 14 days later at explantation. On implantation, HeaTro- 8 maintained its spongy form. HeaTro-2 began to disperse soon after placement in the wound site and became obscured by a thin layer of blood. Split thickness grafts were easily applied over both HeaTro constructs. Macroscopic assessment of the regenerated tissue at explantation following non-grafted treatment with HeaTro-8, HeaTro-2 and to a lesser extent Solosite showed pink blood-containing tissue compared to IDRT treated wounds and open wounds which both appeared white and fibrous. Assessment of split thickness graft (STG) viability at explantation indicated that HeaTro-8 and HeaTro-2 both allowed for plasmatic imbibition and graft take that yielded a similar macroscopic appearance to that of the STG treatment alone.

Histology of full thickness sections through the center of each wound included H\&E, VVG and picrosirius red (PSR) staining to assess local tissue performance, and immunohistochemistry encompassing CD31 and vWF staining to allow for blood vessel quantification. Representative images of $\mathrm{H} \& \mathrm{E}, \mathrm{vWF}, \mathrm{CD} 31$ and polarized PSR staining are shown in Figure 6 (non-grafted samples) and Figure 7 (grafted samples). VVG staining, performed to report on any residual HeaTro revealed that the material had dissipated by 2 weeks (data not shown). Of the non-grafted treatments, HeaTro- 8 implants generated healing wound sites that most resembled normal skin displaying advanced epidermal and dermal regeneration typical of a wound in the maturation phase of repair. The epidermal layers of HeaTro- 8 treated wounds were characterized by rete ridges and keratinizing stratified 


\section{WILEY-VCH}

epithelium. Quantitative analysis of the treatment effects on endothelial markers indicated diminished levels of the endothelial marker CD31 and reduced vWF staining for HeaTro-8 treated wounds. This is consistent with the beneficial effects of an early appearance of functional vasculature that had subsequently returned to normal, as expected for a healing wound that had progressed into the maturation phase ${ }^{[6 \mathrm{~b}]}$. This superior healing was reflected in the polarized PSR HeaTro-8 sections which showed thick, aligned weaving collagen bundles running parallel to the epidermis in the deep-dermis. HeaTro-2 showed less pronounced epidermal and dermal development but this was better than the granulation tissue appearance of Solosite. IDRT healing was developed but heterogeneous, while the open wound presented partially healed tissue with evidence of regenerating dermis and epidermis. The repair of open wounds was helped by STGs. HeaTro-8 and HeaTro-2 both allowed for the viable incorporation of STGs that were concomitantly grafted onto the treated sites on Day 0. These were characterized by organizing collagen, and levels of vascularization comparable to treatment with STG alone.

The ensemble of histology and immunohistochemistry images was then scored based on multiple skin components in order to rank the extent of wound repair (Figure 8). ${ }^{[35]}$ Of the non-grafted treatments HeaTro- 8 ranked as superior on the basis of appearance of the epidermis, cell type, extent of perfused vasculature, and collagen organization. One finding for the epidermis could not be ranked due to its loss in explant handling; if this had been present the average line score would have exceeded the calculated value and would have further highlighted HeaTro-8 as the leading wound repair material. HeaTro-2 and -8 had promoted dermal and epidermal growth, whereas Solosite, IDRT and open wounds all showed slower repair. Granulomatous tissue was evident with Solosite. IDRT persisted over part of its treated sites and presented with fewer blood vessels that projected towards the epidermis. Scoring of the grafted treatments indicated the wounds had undergone comparable healing. 


\section{WILEY-VCH}

\section{Conclusion}

We find that implantation of HeaTro into a full thickness dermal wound bed generates an environment that facilitates accelerated progression through wound healing phases, culminating in regenerated tissue approaching that of unwounded skin. The unique physical and tissue interactive properties of HeaTro products lead to superior repair over leading forms of wound treatment, encompassing hydrogel, dermal regeneration template, and sterile bandaging of open wounds. We conclude that HeaTro is a novel class of biomaterial that promotes tissue repair. These findings establish a foundation for the construction of novel materials that utilize tropoelastin's ability to promote wound healing. 


\section{WILEY-VCH}

\section{Experimental section}

Heat-based formation of stabilized tropoelastin constructs (HeaTro)

Recombinant human tropoelastin isoform SHEL $\Delta 26$ A (synthetic human elastin without domain 26A) corresponding to amino acid residues 27-724 of GenBank entry AAC98394 (gi 182020) was obtained from Elastagen Pty Ltd. ${ }^{[24]}$ For sponge fabrication, tropoelastin $(10 \%$ w/v) was dissolved in $\mathrm{H}_{2} \mathrm{O}$ and pipetted into polydimethylsiloxane (PDMS) molds. Following freezing at $-30^{\circ} \mathrm{C}$ the samples were lyophilized, removed from the molds and placed at $160^{\circ} \mathrm{C}$ for 2-16 h. Characterization of HeaTro was primarily performed on samples heat treated for 8 h. For micropatterned film formation, tropoelastin ( $10 \% \mathrm{w} / \mathrm{v})$ was dissolved in $\mathrm{H}_{2} \mathrm{O}$ and spread over micropatterned PDMS molds. The solution was dried at $37^{\circ} \mathrm{C}$ for $16 \mathrm{~h}$. The resulting films were then placed at $160^{\circ} \mathrm{C}$ for $8 \mathrm{~h}$. For tube formation, tropoelastin $(20 \% \mathrm{w} / \mathrm{v})$ was dissolved in 1,1,1,3,3,3 hexafluoro-2- propanol (HFP) and loaded into a $1 \mathrm{ml}$ syringe connected to a blunt 18-gauge needle and electrospun onto a rotating Teflon-coated stainlesssteel mandrel. The needle was connected to a $15 \mathrm{kV}$ positive power supply and rastered at 10 $\mathrm{cm} / \mathrm{s}$. Tropoelastin solution was ejected through the syringe at a rate of $1 \mathrm{ml} / \mathrm{h}$ using a syringe pump. The rotating (1000 rpm) mandrel was connected to a negative power supply modulated from -4 to $-6 \mathrm{kV}$. The resulting tube was removed from the mandrel and placed at $160^{\circ} \mathrm{C}$ for $16 \mathrm{~h}$

\section{SDS-PAGE analysis}

Following heat stabilization HeaTro samples were hydrated in water for $16 \mathrm{~h}$. Supernatant was removed and boiled for $5 \mathrm{~min}$ in the presence of $\beta$-mercaptoethanol before running on an SDS-PAGE (NuPage 4-12\% Bis-Tris gel, Life Technologies).

\section{Atomic Force Microscopy}




\section{WILEY-VCH}

Surface topography of a HeaTro film was examined using atomic force microscopy (Bruker Multimode VIII). The images were captured at $0.5 \mathrm{~Hz}$ scan rate using soft tapping mode with ultrasharp silicone tip (APP Nano, ACTA-SS-10; resonance frequency 200-400 kHz and force constant $\left.13-77 \mathrm{~N} \mathrm{~m}^{-1}\right)$. For each sample a minimum of 10 images of varied sizes $(5 \times 2.5 \mu \mathrm{m}$, $2 \times 1 \mu \mathrm{m}$, and $1 \times 0.5 \mu \mathrm{m})$ from a minimum of three areas across the samples were recorded. The size and morphology of individual tropoelastin assemblies were resolved from line profiles and 3D images of the sample surface.

\section{Swelling}

The swelling behavior of HeaTro samples was evaluated at $25^{\circ} \mathrm{C}$ in water and PBS. Following heat stabilization, the samples were weighed $\left(\mathrm{W}_{\mathrm{dry}}\right)$, then placed in $5 \mathrm{ml}$ water or PBS for $1 \mathrm{~h}$. For each heat treatment time four samples were tested. Excess liquid was removed from the swelled samples and they were reweighed $\left(\mathrm{W}_{\text {wet }}\right)$. The $\mathrm{g}$ liquid absorbed per $g$ HeaTro was calculated using the equation $\left(\mathrm{W}_{\text {wet }}-\mathrm{W}_{\text {dry }}\right) / \mathrm{W}_{\text {dry. }}$. For water swelling, results were compared statistically using an unpaired t-test; for PBS swelling, results were compared statistically using a one-way analysis of variance (ANOVA) with a Tukey's multiple comparison test.

\section{Mechanical Tests}

HeaTro sponges $(n=4)$ were cast as dog-bone shapes and submerged in phosphate buffered saline (PBS) prior to testing then sprayed with PBS after positioning between clamps. Uniaxial tensile tests of HeaTro sponges $(10 \% \mathrm{w} / \mathrm{v})$ were performed on an Instron 5560 tensile testing machine equipped with a $10 \mathrm{~N}$ capacity load cell. Samples were stretched at a constant strain rate of $254 \mathrm{~mm} / \mathrm{min}$ until break. The elastic modulus was calculated as the tangent slope of the stress-strain curve at $10-40 \%$ strain and data were smoothed using a 10 point rolling average. 


\section{WILEY-VCH}

Amino acid analysis (AAA)

AAA was performed on $20 \mathrm{mg}$ samples of tropoelastin and HeaTro at the Australian Proteome Analysis Facility, Sydney Australia. Dried tropoelastin and HeaTro samples were weighed and subjected to $24 \mathrm{~h}$ liquid hydrolysis in $6 \mathrm{M} \mathrm{HCl}$ at $110^{\circ} \mathrm{C}$. After hydrolysis, amino acids were analyzed using the Waters AccQTagUltra chemistry on a Waters Acquity UPLC.

\section{Mass Spectrometry}

Dimers from 4 different HeaTro samples were compared to tropoelastin monomer. Following SDS-PAGE analysis, gel bands corresponding to untreated tropoelastin monomer and dimer from a HeaTro sample heat-stabilized for $2 \mathrm{~h}$ were excised. In-gel digestion was performed with endoproteinase Lys-C (sequencing grade; Sigma Aldrich) at $37^{\circ} \mathrm{C}$ for $16 \mathrm{~h}$, after which 1 $\mu l$ was removed and mixed with $1 \mu l \alpha$-cyano-4-hydroxycinnamic acid matrix solution and loaded onto a target plate for MALDI-TOF mass spectrometry on a QSTAR Elite mass spectrometer in reflectron mode.

PeptideMass (Expasy Bioinformatics Resource Portal) was used to calculate peptide masses ranging from 500 to $8000 \mathrm{Da}$ including peptides with missed cleavages to account for the possibility of incomplete digestion particularly due to the presence of proline residues following lysine residues, which is known to prevent cleavage. ${ }^{[36]}$ Mass intensities were compared between monomer and dimer on both the raw data and following normalization of the monomer and dimer to 4 different peptides.

\section{Fourier transform infrared (FTIR) Spectroscopy}

Vibrational spectroscopy was performed on dry tropoelastin and HeaTro samples $(\sim 8 \mathrm{mg})$. FTIR spectra were collected using a Bruker IFS66V FTIR spectrometer (Bruker, Karlsruhe, 


\section{WILEY-VCH}

Germany) equipped with a KBr beamsplitter and DLTGS detector. The sampling accessory was a MIRacle single reflection horizontal attenuated total reflectance (ATR) (Pike Technologies, Madison, WI) equipped with a composite diamond internal reflection element (IRE) with a 2-mm sampling surface and a $\mathrm{ZnSe}$ focusing element. Single-beam spectra of the samples were obtained and ratioed against a single-beam background spectrum of air to produce a spectrum in absorbance units. The spectrum of a clean, blank ATR crystal surface was used as reference. After every measurement, the ATR crystal was washed with isopropyl alcohol and air-dried and a new background spectrum was collected. Spectra were collected over the region of $4000-525 \mathrm{~cm}^{-1}$ with the co-addition of 128 scans at a resolution of $4 \mathrm{~cm}^{-1}$. Spectra of each sample were collected in triplicate.

\section{Raman Spectroscopy}

Raman spectra were collected using a Bruker MultiRAM FT-Raman spectrometer (Bruker, Karlsruhe, Germany) equipped with a Nd:YAG laser emitting at $1064 \mathrm{~nm}$ and a liquidnitrogen-cooled germanium detector with an extended spectral region of $4000-50 \mathrm{~cm}^{-1}$. A $180^{\circ}$ back-scattering sampling geometry was employed. Spectral resolution was $4 \mathrm{~cm}^{-1}$ with the co-addition of 100 scans at a laser power of $200 \mathrm{~mW}$. A Blackman-Harris 4 term apodization function was applied. Spectra of each sample were collected in triplicate and the data were averaged.

\section{$N M R$}

Solid-state Cross-Polarization Magic Angle Spinning (MAS) Carbon-13 NMR data were acquired at $9.4 \mathrm{~T}\left(399.9575 \mathrm{MHz}{ }^{1} \mathrm{H}, 100.5789 \mathrm{MHz}{ }^{13} \mathrm{C}\right)$ on a Varian/Agilent Unity Inova WB 400 spectrometer, equipped with a $3.2 \mathrm{~mm}$ HX-T3 MAS probe (Chemagnetics/Varian NMR, Fort Collins, CO). Typical sample sizes for tropoelastin and HeaTro were $11 \mathrm{mg}$. The spinning speed used in MAS experiments was $v_{R}=8 \mathrm{kHz}$. Data were acquired at $22.0^{\circ} \mathrm{C}$. 


\section{WILEY-VCH}

${ }^{13} \mathrm{C}$ chemical shifts were referenced to the tetramethylsilane scale, using hexamethylbenzene as an external standard $\left[\delta\left({ }^{13} \mathrm{CH}_{3}\right)=17.0 \mathrm{ppm}\right]$. Cross-polarization from ${ }^{1} \mathrm{H}$ to ${ }^{13} \mathrm{C}$ was accomplished at a ${ }^{1} \mathrm{H}$ spin-lock field of $\gamma_{\mathrm{H}} B_{1}{ }^{\mathrm{H}} / 2 \pi=42 \mathrm{kHz}$ and ${ }^{13} \mathrm{C}$ spin-lock field of $\gamma_{\mathrm{C}} B_{1}{ }^{\mathrm{C}} / 2 \pi$ $=50 \mathrm{kHz}\left(n=1\right.$ Hartmann-Hahn matching condition, $\left.\gamma_{\mathrm{C}} B_{1}{ }^{\mathrm{C}} / 2 \pi=\gamma_{\mathrm{H}} B_{1}{ }^{\mathrm{H}} / 2 \pi+\nu_{\mathrm{R}}\right)$. A $3.0 \mu \mathrm{s}{ }^{1} \mathrm{H}$ $90^{\circ}$ pulse was followed by $1 \mathrm{~ms}$ contact time and recycle delay was $5 \mathrm{~s}$. An applied ${ }^{1} \mathrm{H}$ field strength for high-power TPPM decoupling ${ }^{[37]}$ during acquisition was $\gamma_{\mathrm{H}} B_{1} / 2 \pi=83 \mathrm{kHz} .3800$ scans were averaged for each sample with an experimental time of $5 \mathrm{~h}$.

Small Angle X-ray Scattering (SAXS) and Wide Angle X-ray scattering (WAXS) Tropoelastin and HeaTro samples $(\sim 8 \mathrm{mg})$ were supplied to the Australian Synchrotron (Clayton, Victoria). Transmission-geometry SAXS measurements were made on each sample, using three different SAXS camera lengths $(0.9 \mathrm{~m}, 2.6 \mathrm{~m}$ and $7 \mathrm{~m})$ and different $\mathrm{x}$-ray wavelengths to cover the widest range of scattering angles possible. The size of the $\mathrm{x}$-ray beam footprint on the sample was $0.25 \times 0.15 \mathrm{~mm}$. Most $\mathrm{x}$-ray exposures were $1 \mathrm{~s}$ with the beam off during translation of the specimen. Data were collected on a Pilatus-2 $1 \mathrm{M}$ area detector. Silver behenate was used as a calibrant for scattering angle and pure water provided an absolute scattering intensity standard. Conversion of all area detector images to onedimensional scattering profiles was done via the software packages ScatterBrain ${ }^{[38]}$ and Saxs15ID. ${ }^{[39]}$

\section{Cell proliferation}

HeaTro sponges were placed on sterilized PDMS molds in 24 well tissue culture plates and secured in place with CellCrown inserts (Sigma). Prior to cell-seeding, the sponges were equilibrated for $16 \mathrm{~h}$ at $37^{\circ} \mathrm{C}$ in Dulbecco's Modified Eagle's Medium (DMEM) containing $10 \%$ fetal bovine serum (FBS) and 1\% penicillin-streptomycin. Human dermal fibroblasts (GM03348; Coriell Institute) were seeded (1.2 x 104 cells/well) onto HeaTro and cultured for 


\section{WILEY-VCH}

up to 7 days at $37^{\circ} \mathrm{C}$ and $5 \% \mathrm{CO}_{2}$. At Days 1,3 and 7 the sponges $(\mathrm{n}=3)$ were peeled off the PDMS molds and moved to another 24 well plate where the cultured cells were trypsinized and collected. Cell proliferation was assessed using a CyQuant Cell Proliferation Assay Kit (Thermo Fisher Scientific) to determine DNA content. The experiment was repeated three times. Results were compared statistically using one-way ANOVA with a Tukey's multiple comparison test.

\section{Fibroblast scratch assay}

Prior to cell culturing, FBS was incubated at $37^{\circ} \mathrm{C}$ for 7 days in the presence or absence of a HeaTro sample stabilized by heating at $160^{\circ} \mathrm{C}$ for $2 \mathrm{~h}$. The FBS was then added to DMEM to form 2 types of media: DMEM + 10\% FBS + 1\% penicillin-streptomycin (DMEM + FBS) and DMEM + 10\% FBS containing solubilized HeaTro + 1\% penicillin-streptomycin $\left(\right.$ DMEM + FBS + HeaTro). Human dermal fibroblasts (GM03348) were seeded $\left(1 \times 10^{4}\right.$ cells/well) into 6-well glass bottom plates (Part No P06G-1.5-14-F; Mattek). Three wells were cultured in DMEM + FBS and three wells were cultured in DMEM + FBS + HeaTro. Media were changed every 2 days. After four days in culture, a scratch was introduced across the cells using a $20 \mu 1$ sterile pipette tip. Live cell imaging on a Nikon Ti-E Spinning Disk Confocal Live Cell microscope monitored fibroblast movement over the following $22 \mathrm{~h}$. Three positions in each well were observed and images were taken every $15 \mathrm{~min}$. For each position, the scratch width at three sites was measured. Scratch closure at 27 sites was measured for each treatment. The percent scratch remaining was calculated at $0,150,300$, $450,600,750,900,1050$ and $1200 \mathrm{~min}$. The average rate of cell movement was calculated for the two treatments (scratch width was $475 \mu \mathrm{m}$ for DMEM + FBS and $550 \mu \mathrm{m}$ for DMEM + FBS + HeaTro). Results were compared statistically using an unpaired t-test.

\section{HeaTro stability}




\section{WILEY-VCH}

HeaTro samples $\left(15 \mathrm{mg}\right.$ ) were stabilized for $2,4,6,8$ or $10 \mathrm{~h}$ at $160^{\circ} \mathrm{C}$, and placed in $100 \%$ FBS $(n=3)$ at $37^{\circ} \mathrm{C}$ for 7 days. Samples were washed three times in $\mathrm{H}_{2} \mathrm{O}$ for $10 \mathrm{~min}$, lyophilized and weighed. The extent of HeaTro solubilization in FBS was assessed by comparing the final and starting masses.

\section{Mouse model}

A murine full thickness wound model with autografting was used to assess HeaTro stability and biocompatibility in vivo. This study was conducted at the ANZAC Research Institute, NSW Australia and was approved by the SLHD Animal Welfare Committee (AWC 2013/019B) under the Australian National Health and Medical Research Council guidelines for animal experimentation. Four square full thickness skin wounds $(1 \times 1 \mathrm{~cm})$ were made on the back of 12 mice (Balb/c, male) at 10 weeks of age. Explanted skin pieces were used as autografts over the test samples. On each mouse two $8 \mathrm{mg}$ HeaTro constructs of either HeaTro-2, -4, -6 or -8 and an Integra Dermal Regeneration Template (IDRT; Integra Life Sciences Corporation, Plainsboro, NJ) sample (all $0.8 \mathrm{~cm}$ diameter) were applied to three wound sites and autografted. The fourth wound site was left as an open wound. Positioning of the treatments was randomized. Two weeks after implantation, the mice were sacrificed and skin tissue at the site of the wounds was explanted, fixed in $10 \%$ formalin for $24 \mathrm{~h}$ then paraffin embedded, sectioned and stained with hematoxylin and eosin (H\&E) and Verhoeff van Gieson (VVG) stains. Stained section images were acquired with an Olympus VS120 Slide Scanner and OlyVIA software.

\section{Pig model}

A porcine full thickness wound model was used to assess the effect of two HeaTro formulations on dermal wound healing. This study was conducted in the School of Animal and Veterinary Sciences at Charles Sturt University, NSW Australia and was approved by the 


\section{WILEY-VCH}

Animal Care and Ethics Committee (ACEC 15/117). Nine replicates of eight sample types were applied to six wound sites across twelve pigs (Camborough-29 female, $\sim 3$-month-old gilt): HeaTro stabilized for 2 or $8 \mathrm{~h}$ with and without a split thickness graft, open wound, split thickness graft, IDRT with the silicone layer removed and Solosite (Smith \& Nephew). Following anesthesia six wound sites (each $2 \mathrm{~cm} \times 2 \mathrm{~cm}$ and arrayed on each side of the midline: two cranial, two mid and two caudal wounds) were created on the dorsum of each pig. When required, split thickness grafts were harvested from the flank of the same pig undergoing surgery using a power-driven dermatome set at a thickness of $0.3 \mathrm{~mm}$. The grafts were held in place with a suture at the four corners of each wound. Following treatment each wound was dressed with Opsite Post-Op waterproof dressing (6.5 x 5 cm; Smith \& Nephew) followed by bandaging and then a body wrap of an elasticated dressing (Vetwrap). Analgesia and antibiotics were provided for the first four post-operative days and a non-steroidal analgesic was administered for a further five days. Dressing changes were performed four and seven days post-surgery. Fourteen days after treatment, the pigs were euthanized where it was noted that in some cases the healing of the caudal and mid-line wound sites had been compromised by self-trauma; in contrast, the cranial wound sites had been allowed to heal without any external physical interference. Because of the potential that the caudal and midline wounds had been affected by physical irritation by the pigs, and the difficulty and potential bias involved in trying to select and disregard data from traumatized sites, quantitative analysis focused on dermal integrity, epidermal repair and maturation of the healed wounds at the three cranial sites for each sample type (24 sites in total across 12 pigs; HeaTro-8, HeaTro-2, IDRT, Solosite, open, HeaTro-8 + STG, HeaTro-2 + STG and STG). Wound sites were excised with a margin of surrounding normal skin for histological examination. Three unwounded normal skin samples were also excised for comparison. Histology sections were cut midway through the explanted skin, parallel to the cranial edge, paraffin embedded, sectioned and stained with H\&E, VVG and PicroSirius Red (PSR). 


\section{WILEY-VCH}

Immunohistochemical staining was also performed with anti-von Willebrand Factor (Dako, IR52761-2, FLEX Polyclonal RB) and anti-CD31 (Abcam, ab28364, Lot No: GR244952, GR272058) antibodies and detected using an Envision FLEX mini Kit, High pH (Dako, K802421-2). Stained section images were acquired with an Olympus VS120 Slide Scanner and OlyVIA software. For each, immuno-stained section three equally sized regions were analyzed from the upper, mid and deep dermis. FIJI software was used to quantify $10-100 \mu \mathrm{m}$ diameter DAB-stained blood vessel numbers. Results were compared statistically using twoway ANOVA with a Tukey's multiple comparison test.

The ensemble of histology and immunohistochemistry images was then scored based on the literature. ${ }^{[35]}$ On this basis, the following scores were allocated to multiple skin components in order to rank the extent of wound repair. Epidermis: 1 - sides only/discontinuous, 2 substantial/flat, 3 - mature with rete ridges. Cell type: 1 - lymphocyte-rich throughout, 2 elevated lymphocytes confined to upper dermis, 3 - predominantly macrophage and fibroblast rich. Perfused blood vessels: 1 - elevated throughout, 2 - elevated in upper dermis, 3 regressed. Collagen organization: 1 - chaotic/disorganized throughout, 2 - deep-dermal organization, 3 - aligning across wound/intercalating at wound edge.

\section{Statistical Analysis}

Graph Pad Prism version 6.07 software was used for statistical analysis. Student's unpaired ttests were used for water swelling $(n=4)$ and fibroblast migration $(n=3)$ data analysis. Oneway ANOVA with a Tukey's multiple comparison test was used to assess PBS swelling $(n=4)$ and cell proliferation $(n=3)$ data. Two-way ANOVA with a Tukey's multiple comparison test was used to assess endothelial marker data $(n=3)$. Statistical significance was accepted at values of $\mathrm{P}<0.05$ and indicated in the figures by asterisks $(* \mathrm{P}<0.05, * * \mathrm{P}<0.01, * * * \mathrm{P}<0.001$, $* * * * \mathrm{P}<0.0001)$. Data are presented as mean $\pm \mathrm{SEM}$. 


\section{WILEY-VCH}

\section{Supporting Information}

Supporting Information is available from the Wiley Online Library or from the author.

\section{Acknowledgements}

We acknowledge support from the Wellcome Trust, Australian Research Council, National Health \& Medical Research Council and Elastagen Pty Ltd. We thank Seonaid Grimmer for invaluable assistance with the pig studies, Edwin Brackenreg with preliminary analysis and the sample preparation for NMR, Ali Fathi with Instron studies, Sanaz Maleki with histology and Michael Kuligowski with microscopy. The authors acknowledge the facilities and the scientific and technical assistance of the Australian Microscopy \& Microanalysis Research Facility at the Australian Centre for Microscopy \& Microanalysis at the University of Sydney, and Isabelle Meyer-Carrive and Edmund Ho at ICP Firefly Pty Ltd. Access to the Australian Synchrotron (ANSTO) was provided by the NSW Industry Synchrotron Access Scheme. ASW is the Scientific Founder of Elastagen Pty Ltd.

Received: ((will be filled in by the editorial staff))

Revised: ((will be filled in by the editorial staff)) Published online: ((will be filled in by the editorial staff)) 


\section{WILEY-VCH}

References

[1] WHO, Burns, http://www.who.int/mediacentre/factsheets/fs365/en/, accessed: 04/04/2017, 2017.

[2] K. Jarbrink, G. Ni, H. Sonnergren, A. Schmidtchen, C. Pang, R. Bajpai, J. Car, Systematic reviews 2016, 5, 152.

[3] C. K. Sen, G. M. Gordillo, S. Roy, R. Kirsner, L. Lambert, T. K. Hunt, F. Gottrup, G. C. Gurtner, M. T. Longaker, Wound repair and regeneration : official publication of the Wound Healing Society [and] the European Tissue Repair Society 2009, 17, 763.

[4] National Evidence-Based Guideline on Prevention Identification and Management of Foot Complications in Diabetes, Part of the Guidelines on Management of Type 2 Diabetes, https://www.baker.edu.au/Assets/Files/Foot_FullGuideline_23062011.pdf, accessed: 13 Feb, 2017.

[5] G. C. Gurtner, S. Werner, Y. Barrandon, M. T. Longaker, Nature 2008, 453, 314.

[6] a) P. Martin, Science (New York, N.Y.) 1997, 276, 75; b) J. M. Reinke, H. Sorg, European surgical research. Europaische chirurgische Forschung. Recherches chirurgicales europeennes 2012, 49, 35; c) D. G. Simpson, Expert review of medical devices 2006, 3, 471; d) J. F. Almine, S. G. Wise, A. S. Weiss, Birth defects research. Part C, Embryo today : reviews 2012, 96, 248.

[7] S. Das, A. B. Baker, Frontiers in bioengineering and biotechnology 2016, 4, 82.

[8] J. F. Almine, S. G. Wise, M. Hiob, N. K. Singh, K. K. Tiwari, S. Vali, T. Abbasi, A. S. Weiss, FASEB journal : official publication of the Federation of American Societies for Experimental Biology 2013, 27, 3455.

[9] a) D. V. Bax, U. R. Rodgers, M. M. Bilek, A. S. Weiss, The Journal of biological chemistry 2009, 284, 28616; b) J. Rnjak, Z. Li, P. K. Maitz, S. G. Wise, A. S. Weiss, Biomaterials 2009, 30, 6469; c) P. Lee, D. V. Bax, M. M. Bilek, A. S. Weiss, The Journal of biological chemistry 2014, 289, 1467; d) P. Lee, G. C. Yeo, A. S. Weiss, The FEBS journal 2017, 284, 2216.

[10] Y. Yu, S. G. Wise, P. L. Michael, D. V. Bax, G. S. Yuen, M. A. Hiob, G. C. Yeo, E. C. Filipe, L. L. Dunn, K. H. Chan, H. Hajian, D. S. Celermajer, A. S. Weiss, M. K. Ng, PloS one 2015, 10, e0131101.

[11] P. C. Brooks, R. A. Clark, D. A. Cheresh, Science (New York, N.Y.) 1994, $264,569$.

[12] I. Kondyurina, S. G. Wise, A. K. Y. Ngo, E. C. Filipe, A. Kondyurin, A. S. Weiss, S. Bao, M. M. M. Bilek, Biomed Mater 2017, 12, 045002.

[13] M. A. Hiob, A. E. Trane, S. G. Wise, P. N. Bernatchez, A. S. Weiss, Nanomedicine (Lond) 2016, 11, 1591.

[14] a) Y. Wang, S. M. Mithieux, Y. Kong, X. Q. Wang, C. Chong, A. Fathi, F. Dehghani, E. Panas, J. Kemnitzer, R. Daniels, R. M. Kimble, P. K. Maitz, Z. Li, A. S. Weiss, Advanced healthcare materials 2015, 4, 577; b) S. Landau, A. A. Szklanny, G. C. Yeo, Y. Shandalov, E. Kosobrodova, A. S. Weiss, S. Levenberg, Biomaterials 2017, 122, 72.

[15] a) N. Annabi, S. M. Mithieux, P. Zorlutuna, G. Camci-Unal, A. S. Weiss, A. Khademhosseini, Biomaterials 2013, 34, 5496; b) N. Annabi, K. Tsang, S. M. Mithieux, M. Nikkhah, A. Ameri, A. Khademhosseini, A. S. Weiss, Advanced functional materials 2013, 23, 4950; c) S. M. Mithieux, J. E. Rasko, A. S. Weiss, Biomaterials 2004, 25, 4921; d) N. Annabi, S. M. Mithieux, A. S. Weiss, F. Dehghani, Biomaterials 2010, 31, 1655.

[16] J. J. Green, J. H. Elisseeff, Nature 2016, 540, 386.

[17] a) I. V. Yannas, A. V. Tobolsky, Nature 1967, 215, 509; b) H. Tsujimoto, A. Tanzawa, H. Miyamoto, T. Horii, M. Tsuji, A. Kawasumi, A. Tamura, Z. Wang, R. Abe, S. Tanaka, K. Yamanaka, M. Matoba, H. Torii, Y. Ozamoto, H. Takamori, S. Suzuki, S. 


\section{WILEY-VCH}

Morita, Y. Ikada, A. Hagiwara, Journal of biomedical materials research. Part B, Applied biomaterials 2015, 103, 1511.

[18] a) J. W. Drexler, H. M. Powell, Tissue engineering. Part C, Methods 2011, 17, 9; b) M. Madaghiele, E. Calo, L. Salvatore, V. Bonfrate, D. Pedone, M. Frigione, A. Sannino, Journal of biomedical materials research. Part A 2016, 104, 186.

[19] A. Varesano, C. Vineis, C. Tonetti, D. O. Sánchez Ramírez, G. Mazzuchetti, Journal of Applied Polymer Science 2014, 131, 40532.

[20] a) T. D. Sutherland, J. S. Church, X. Hu, M. G. Huson, D. L. Kaplan, S. Weisman, PloS one 2011, 6, e16489; b) M. G. Huson, J. S. Church, J. M. Poole, S. Weisman, A. Sriskantha, A. C. Warden, P. M. Campbell, J. A. Ramshaw, T. D. Sutherland, PloS one 2012, 7, e52308.

[21] P. J. Flory, J. R. Jr., The Journal of Chemical Physics 1943, 11, 521.

[22] S. M. Mithieux, S. G. Wise, M. J. Raftery, B. Starcher, A. S. Weiss, Journal of structural biology 2005, 149, 282.

[23] R. S. Asquith, M. S. Otterburn, W. J. Sinclair, Angewandte Chemie International Edition in English 1974, 13, 514.

[24] W. J. Wu, B. Vrhovski, A. S. Weiss, The Journal of biological chemistry 1999, 274, 21719.

[25] S. G. Wise, S. M. Mithieux, M. J. Raftery, A. S. Weiss, Journal of structural biology 2005, 149, 273.

[26] C. Baldock, A. F. Oberhauser, L. Ma, D. Lammie, V. Siegler, S. M. Mithieux, Y. Tu, J. Y. Chow, F. Suleman, M. Malfois, S. Rogers, L. Guo, T. C. Irving, T. J. Wess, A. S. Weiss, Proc Natl Acad Sci U S A 2011, 108, 4322.

[27] Y. C. Fung, Biomechanics - mechanical properties of living tissues, Springer, New York 1993.

[28] L. Debelle, A. J. Alix, S. M. Wei, M. P. Jacob, J. P. Huvenne, M. Berjot, P. Legrand, European journal of biochemistry 1998, 258, 533.

[29] L. Ali, E. M. Green, R. E. Ellis, D. A. Bradley, J. G. Grossmann, C. P. Winlove, Radiation Physics and Chemistry 2004, 71, 951.

[30] E. M. Green, J. C. Mansfield, J. S. Bell, C. P. Winlove, Interface focus 2014, 4, 20130058.

[31] S. M. Mithieux, S. G. Wise, A. S. Weiss, Advanced drug delivery reviews 2013, 65, 421.

[32] a) Z. Indik, W. R. Abrams, U. Kucich, C. W. Gibson, R. P. Mecham, J. Rosenbloom, Archives of biochemistry and biophysics 1990, 280, 80; b) B. D. Wilson, C. C. Gibson, L. K. Sorensen, M. Y. Guilhermier, M. Clinger, L. L. Kelley, Y. T. Shiu, D. Y. Li, Annals of biomedical engineering 2011, 39, 337.

[33] C.-C. Liang, A. Y. Park, J.-L. Guan, Nat. Protocols 2007, 2, 329.

[34] A. Summerfield, F. Meurens, M. E. Ricklin, Molecular Immunology 2015, 66, 14.

[35] a) A. Gupta, P. Kumar, Plastic and Aesthetic Research 2015, 2, 239; b) L. BraimanWiksman, I. Solomonik, R. Spira, T. Tennenbaum, Toxicologic pathology 2007, 35, 767; c) P. Gal, R. Kilik, M. Mokry, B. Vidinsky, T. Vasilenko, S. Mozes, N. Bobrov, Z. Tomori, J. Bober, P. Dvm, Veterinarni Medicina 2008, 53, 652.

[36] P. Giansanti, L. Tsiatsiani, T. Y. Low, A. J. Heck, Nature protocols 2016, 11, 993.

[37] A. E. Bennett, C. M. Rienstra, M. Auger, K. V. Lakshmi, R. G. Griffin, The Journal of Chemical Physics 1995, 103, 6951.

[38] S. Mudie, ScatterBrain, http://www.synchrotron.org.au/aussyncbeamlines/saxswaxs/software-saxswaxs, accessed: May 1, 2017.

[39] D. J. Cookson, Saxs15ID — software for acquiring, processing and viewing SAXS/WAXS image data at ChemMatCARS, 
WILEY-VCH

http://www.synchrotron.org.au/images/beamlines/saxswaxs/saxs15id_manual.pdf, accessed. 


\section{WILEY-VCH}

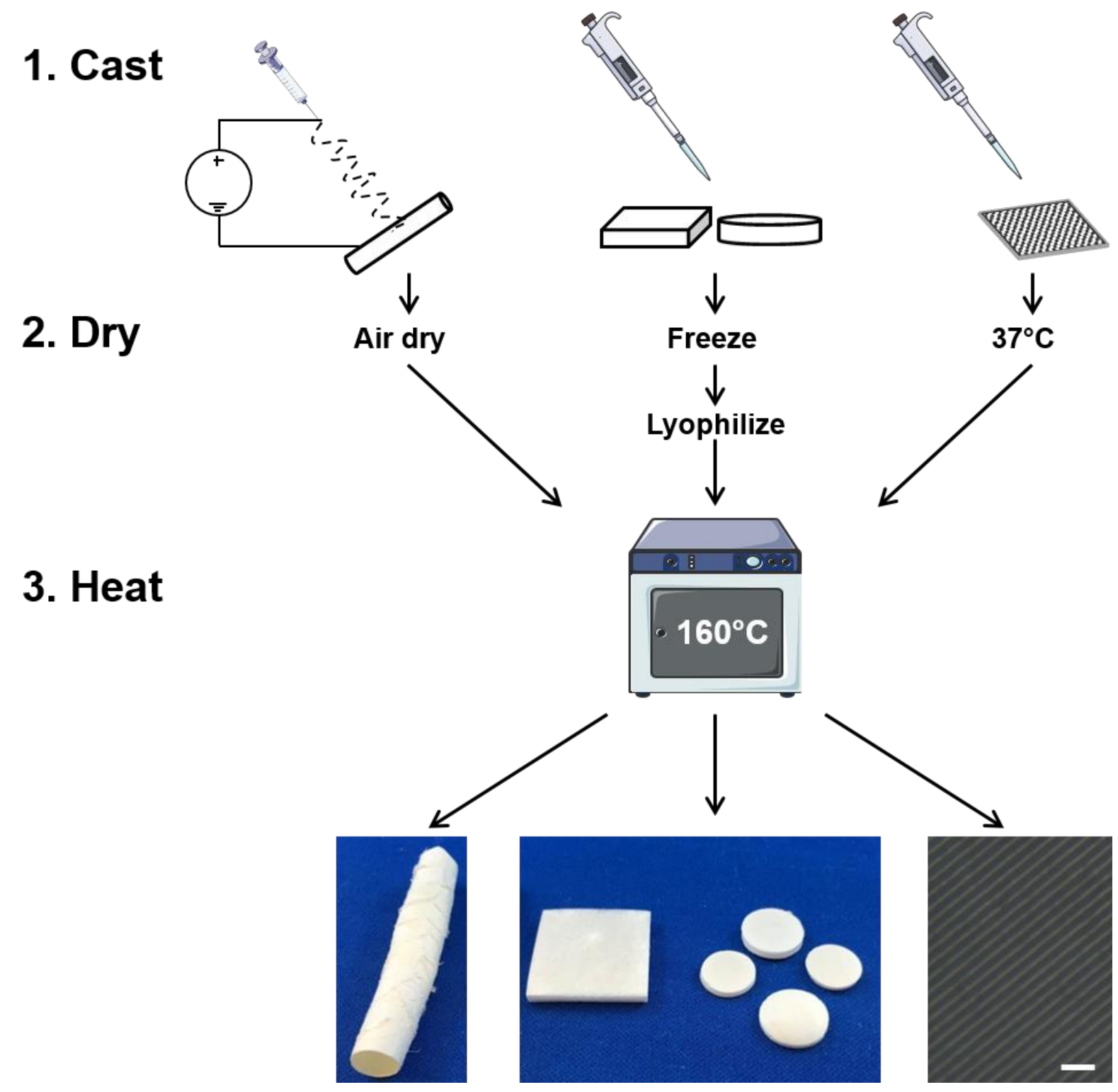

Figure 1. HeaTro fabrication. Following delivery or casting, tropoelastin is heat-stabilized to form shaped and micropatterned constructs. Scale bar is $10 \mu \mathrm{m}$ in the light microscope image of the micropatterned film. 


\section{WILEY-VCH}

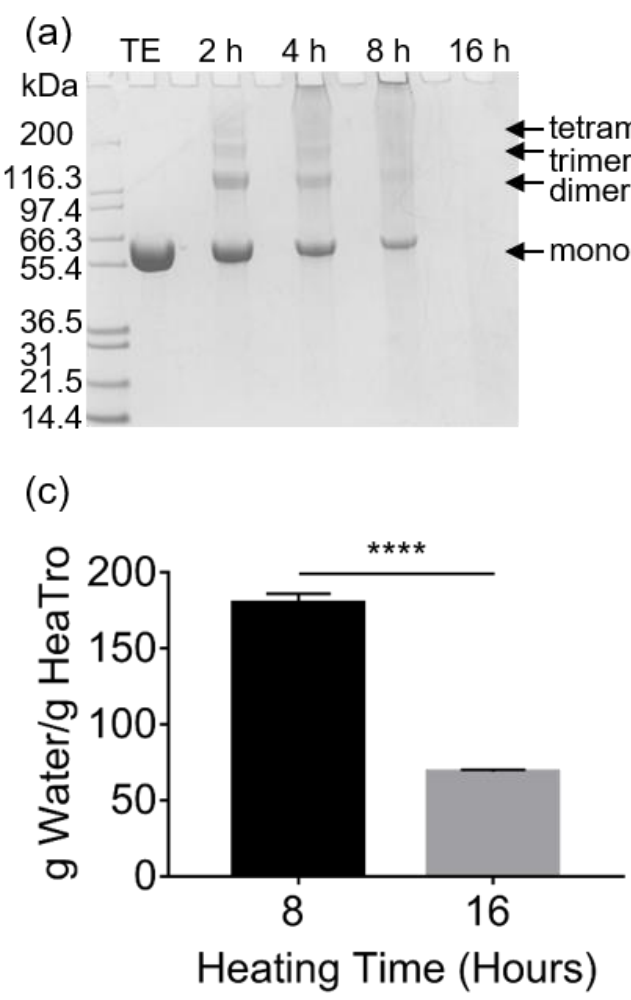

(e)

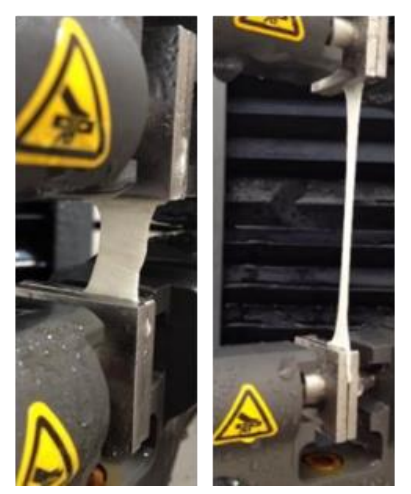

(b)

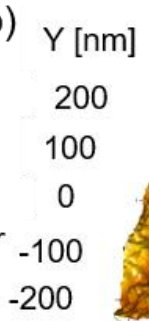

(d)

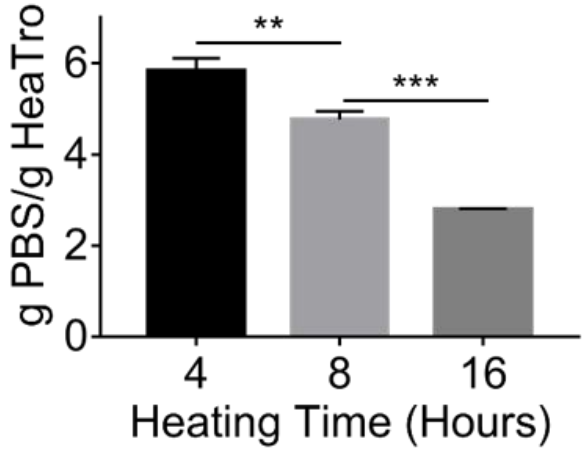

(f)

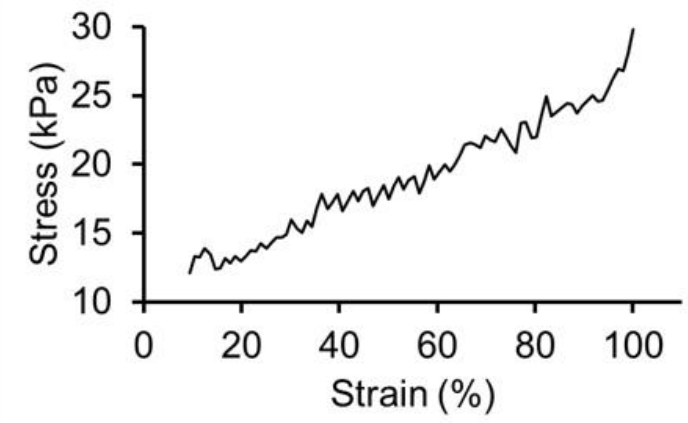

Figure 2. Features of heat-stabilized tropoelastin. (a) SDS-PAGE showing tropoelastin monomer (TE) and tropoelastin-based species from HeaTro constructs that were stabilized for 2, 4,8 or $16 \mathrm{~h}$ at $160^{\circ} \mathrm{C}$. (b) AFM image depicting surface topology of HeaTro. Swelling of HeaTro constructs $(n=4)$ in (c) water, (d) PBS. Data presented as mean \pm SEM, $n=4$. P-values were calculated using an unpaired t-test for water swelling and a one-way ANOVA with a Tukey's multiple comparison test for PBS swelling, $* * \mathrm{P}<0.01, * * * \mathrm{P}<0.001$; **** $\mathrm{P}<0.0001$. HeaTro (e) unstretched and stretched to demonstrate elasticity. (f) Representative stress-strain curve of HeaTro. 
(a)
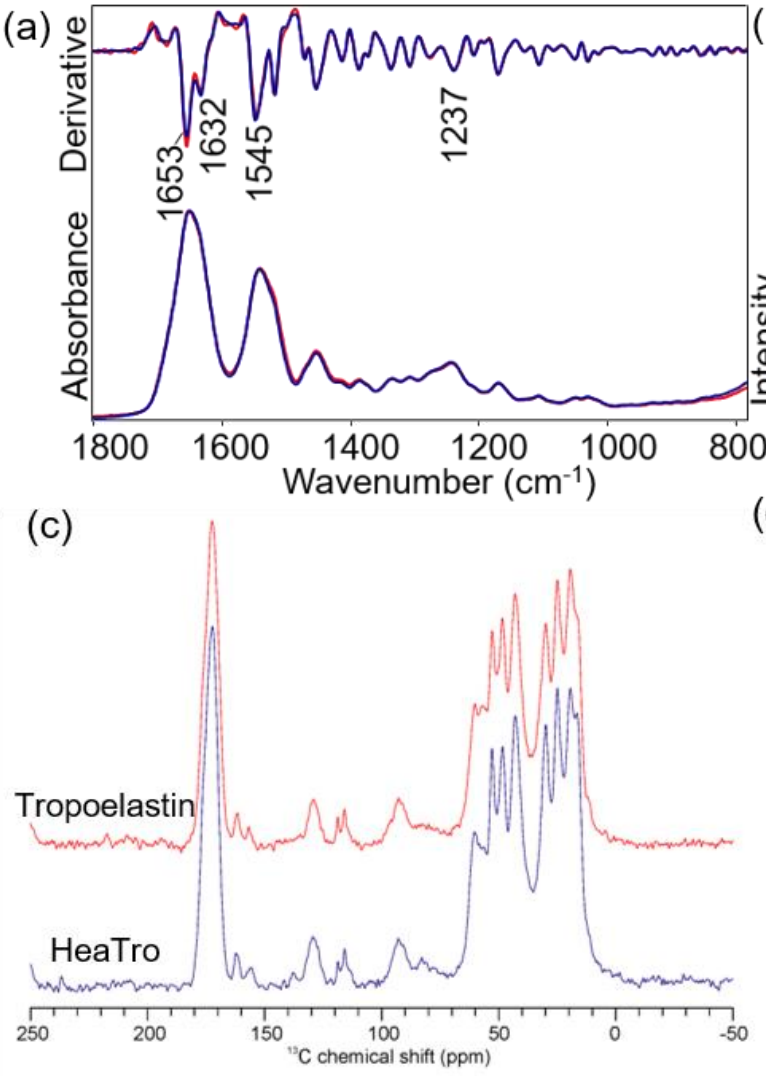

(d)
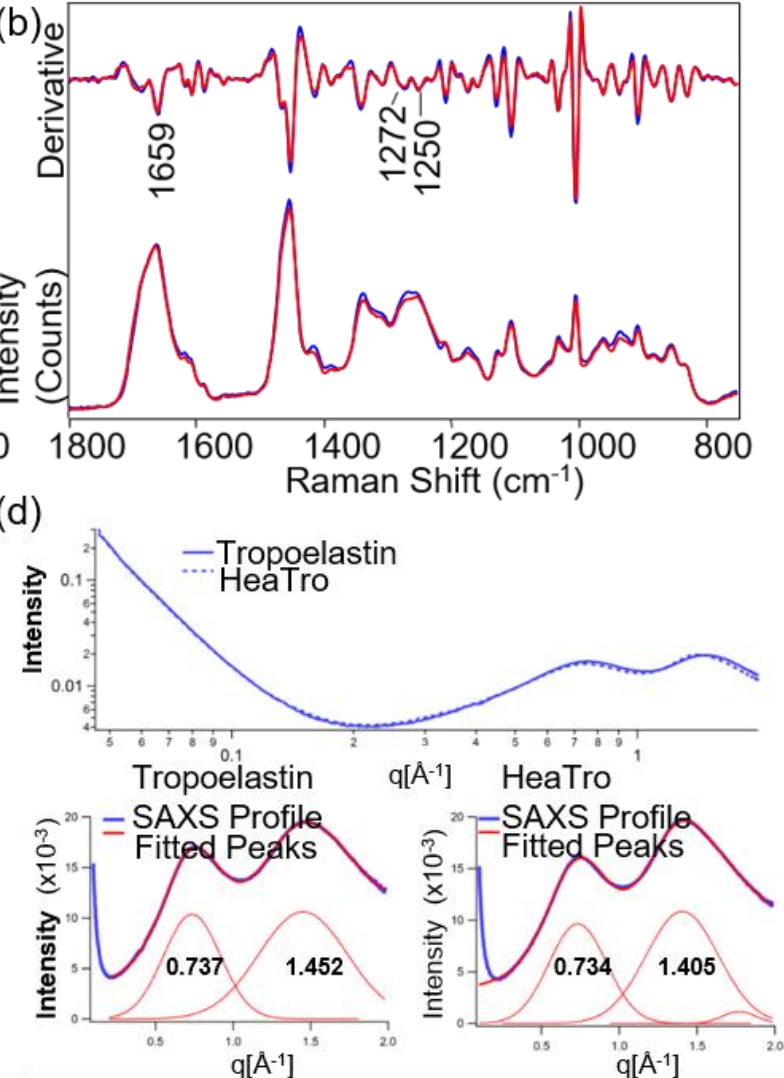

Figure 3. Effect of heat stabilization on tropoelastin secondary structure. (a) FTIR and (b) FT-Raman spectra and corresponding second derivative spectra collected from tropoelastin (red) and HeaTro (blue) showing Amide band positions. (c) Solid-state cross-polarization magic angle spinning carbon-13 NMR spectra of tropoelastin (red) and HeaTro (blue). (d) Small angle $\mathrm{x}$-ray scattering from tropoelastin and HeaTro. Lower graphs present the highestq regions with linear q-scales and Gaussian fitted peaks. 


\section{WILEY-VCH}

(a)

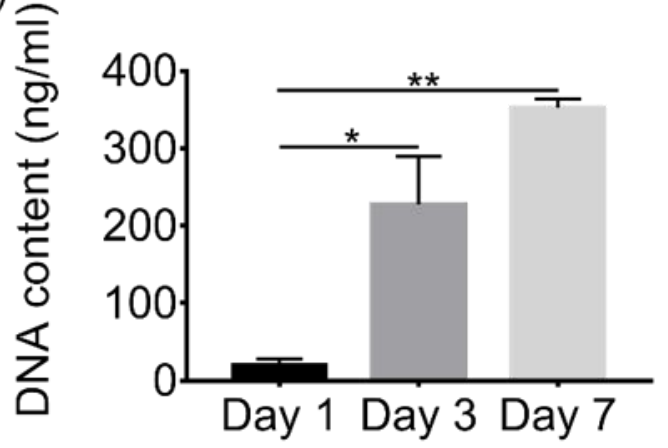

(c)

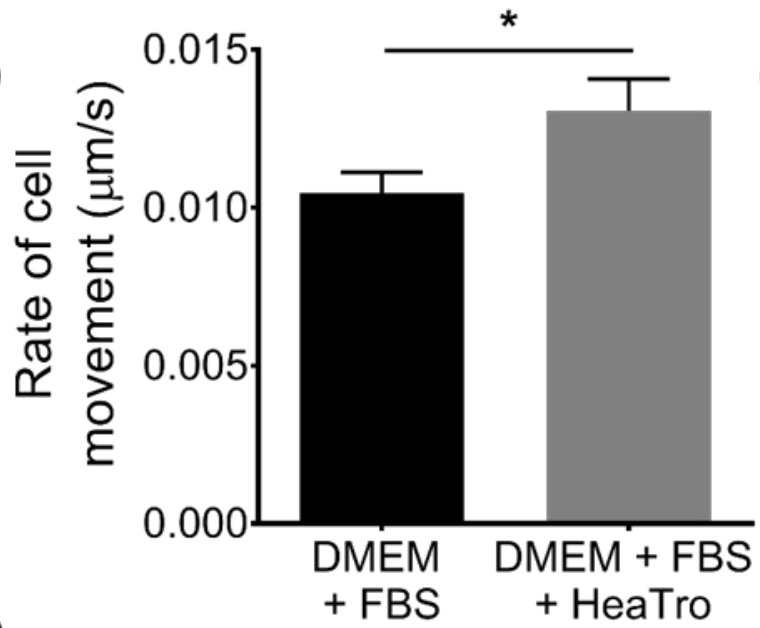

(e)

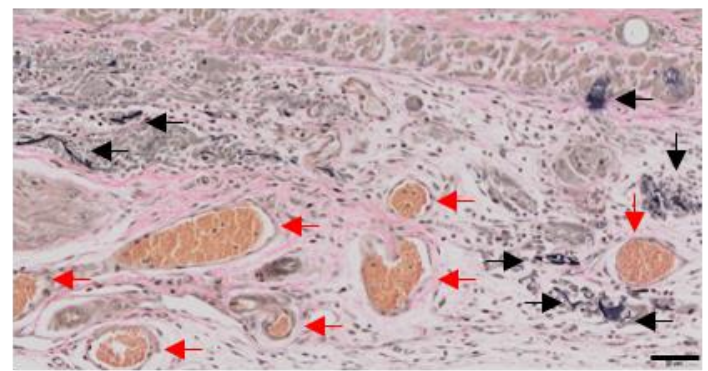

(b)

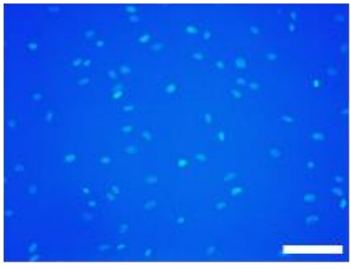

Day 1

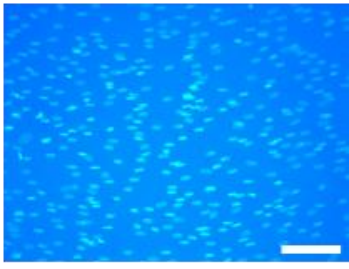

Day 7 (d)

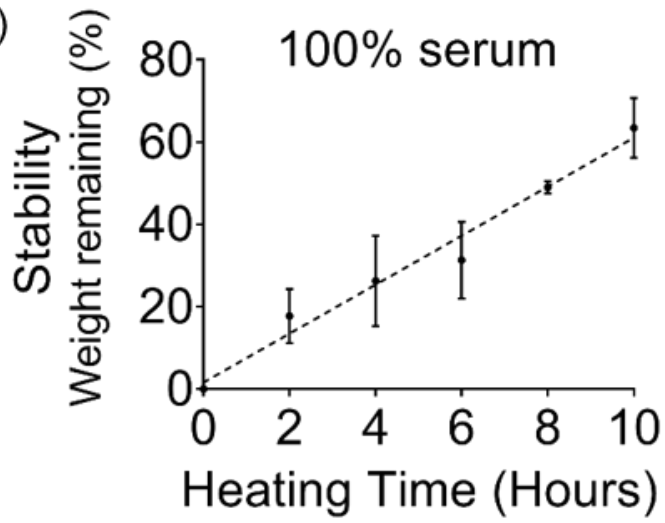

(f)

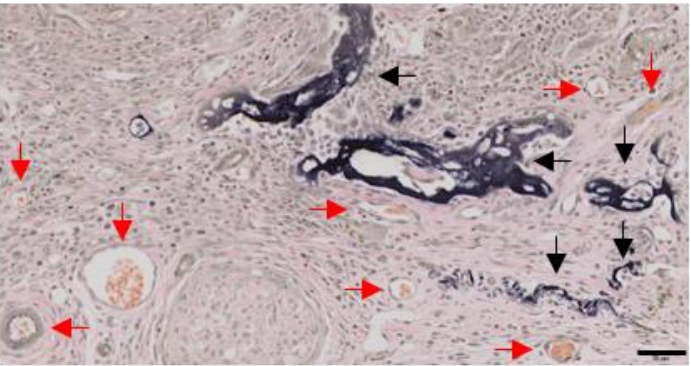

Figure 4. Cell interaction and persistence studies. (a) Fibroblast proliferation on HeaTro over 7 days assessed by total DNA content. Data presented as mean \pm SEM, $n=3$. P-values were calculated using one-way ANOVA with a Tukey's multiple comparison test, ${ }^{*} \mathrm{P}<0.05$, **P $<0.01$. (b) Hoechst-stained fibroblasts on the surface of HeaTro constructs 1 and 7 days post-seeding; scale bar is $100 \mu \mathrm{m}$. (c) Rate of fibroblast migration across a scratch in an in vitro wound healing assay conducted in the absence or presence of solubilized HeaTro. Data presented as mean \pm SEM, $n=3$. P-values were calculated using an unpaired t-test, $* \mathrm{P}<0.05$. In vitro persistence of HeaTro following incubation in fetal bovine serum for 7 days at $37^{\circ} \mathrm{C}$ 


\section{WILEY-VCH}

where HeaTro stability is proportional to heating time ( $\mathrm{d} ; \mathrm{n}=3, \mathrm{R}-\mathrm{sq} \mathrm{0.98)}$. In vivo persistence of HeaTro in a murine full-thickness dermal wound model with autografting. VVG-stained sections from mouse explants 14 days after implantation and autografting of HeaTro constructs stabilized for (e) 6 and (f) $8 \mathrm{~h}$ at $160^{\circ} \mathrm{C}$. Black arrows point to HeaTro; red arrows to perfused blood vessels. Scale bar is $50 \mu \mathrm{m}$. 


\section{WILEY-VCH}

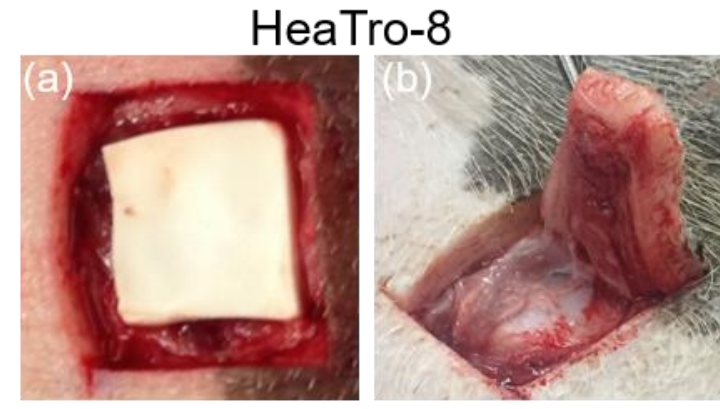

HeaTro-2
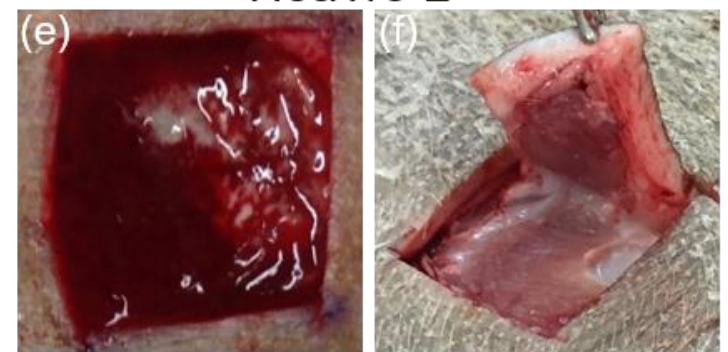

Solosite

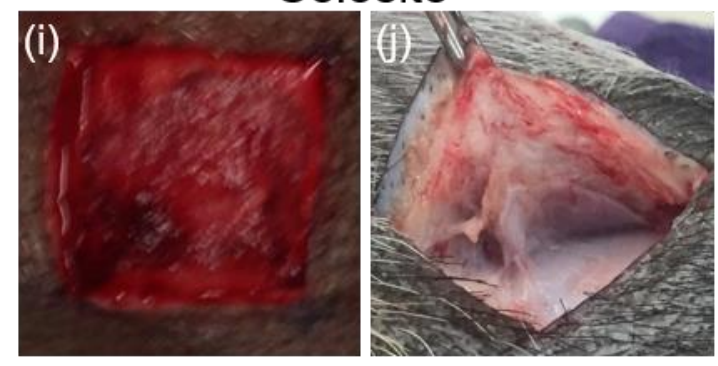

IDRT
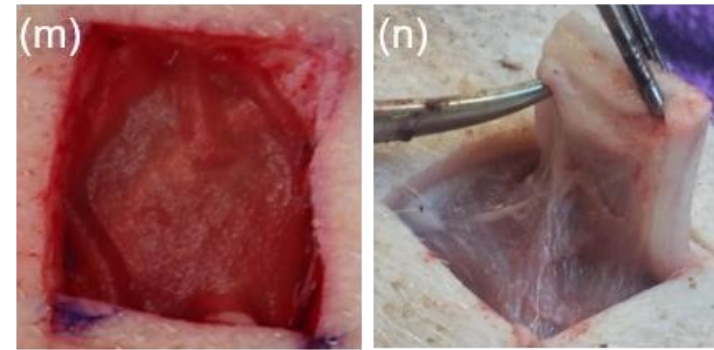

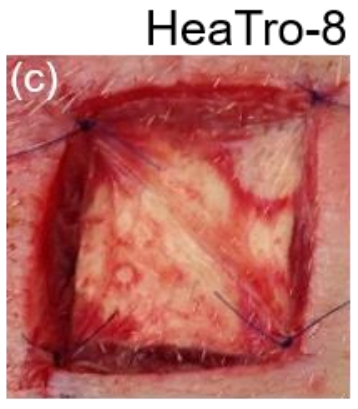

HeaTro-2 + STG

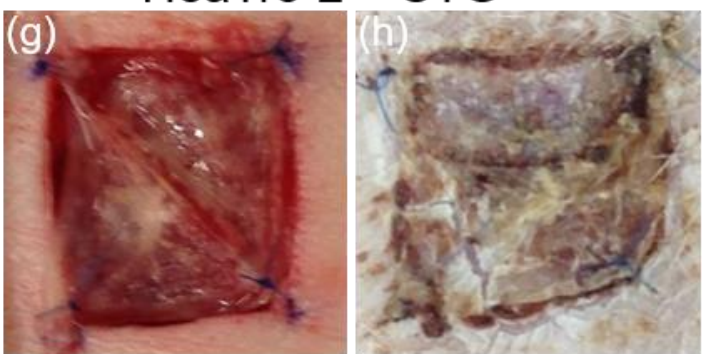

STG

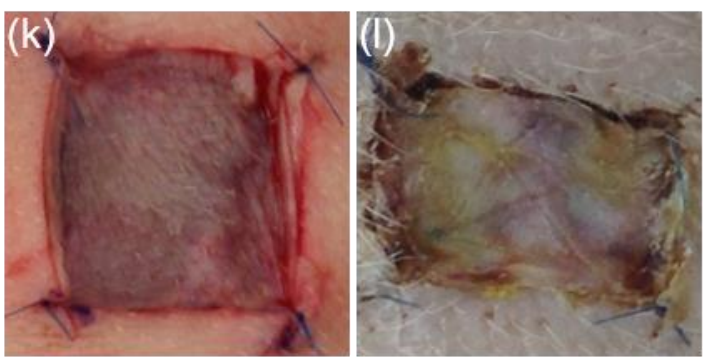

Open wound
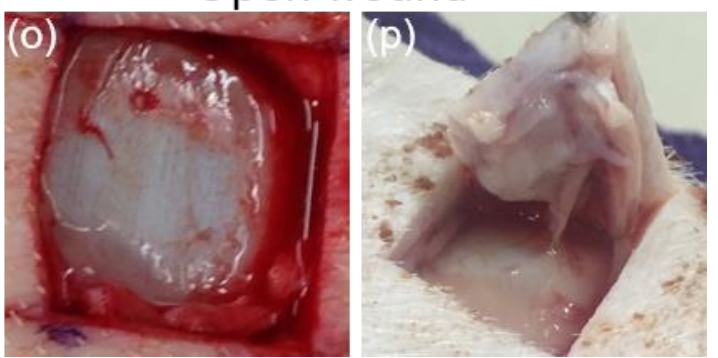

Figure 5. Porcine full-thickness dermal wound repair. Macroscopic images of wounds at the time of implantation (left) and explantation (right)14 days after treatment with (a, b) HeaTro8, (c, d) HeaTro-8 with a split thickness graft, (e, f) HeaTro-2, (g, h) HeaTro-2 with a split thickness graft, (i, j) Solosite, $(k, 1)$ split thickness graft, $(m, n)$ Integra Dermal Regeneration Template, (o,p) open wound. 


\section{WILEY-VCH}

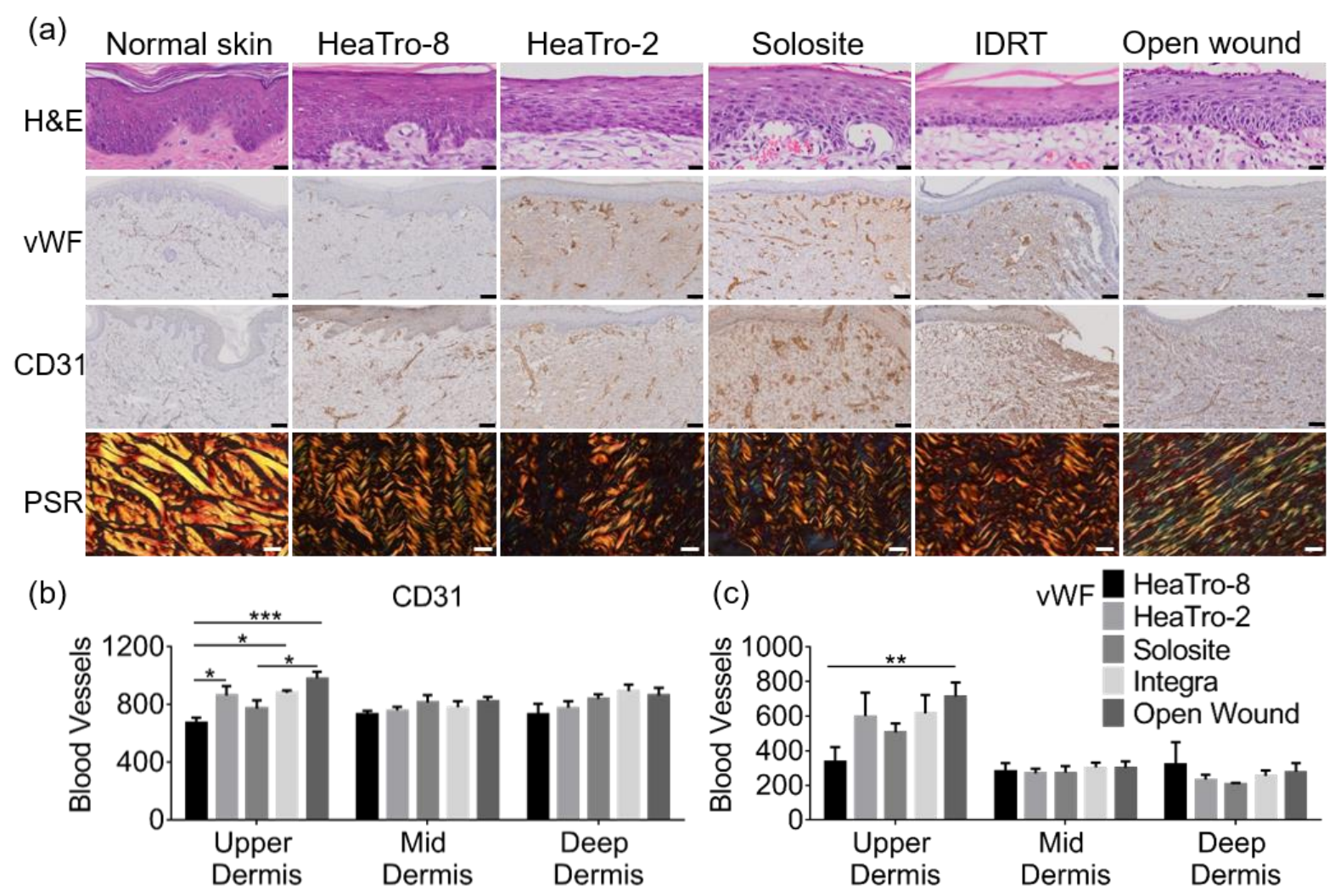

Figure 6. Histology of normal skin and non-grafted pig wounds 14 days after treatment with

HeaTro-8, HeaTro-2, Solosite, Integra Dermal Regeneration Template or open wound. (a)

Representative images of porcine skin sections stained with H\&E (scale bar is $20 \mu \mathrm{m}$ ), antivWF antibody, anti-CD31 antibody (scale bar is $100 \mu \mathrm{m}$ ), and polarized light PSR (scale bar is $20 \mu \mathrm{m})$. Vascularization of non-grafted wounds in the upper, mid and deep-dermis. Blood vessel (10-100 $\mu$ m diameter) numbers calculated using (b) CD31, (c) vWF staining. Data presented as mean \pm SEM, $n=3$. P-values were calculated using two-way ANOVA with a Tukey's multiple comparison test, ${ }^{*} \mathrm{P}<0.05$, $* * \mathrm{P}<0.01$, $* * * \mathrm{P}<0.001$. 


\section{WILEY-VCH}

(a) HeaTro-8 HeaTro-2

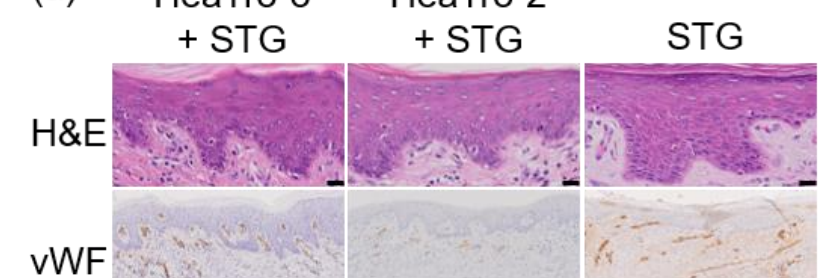

(b)
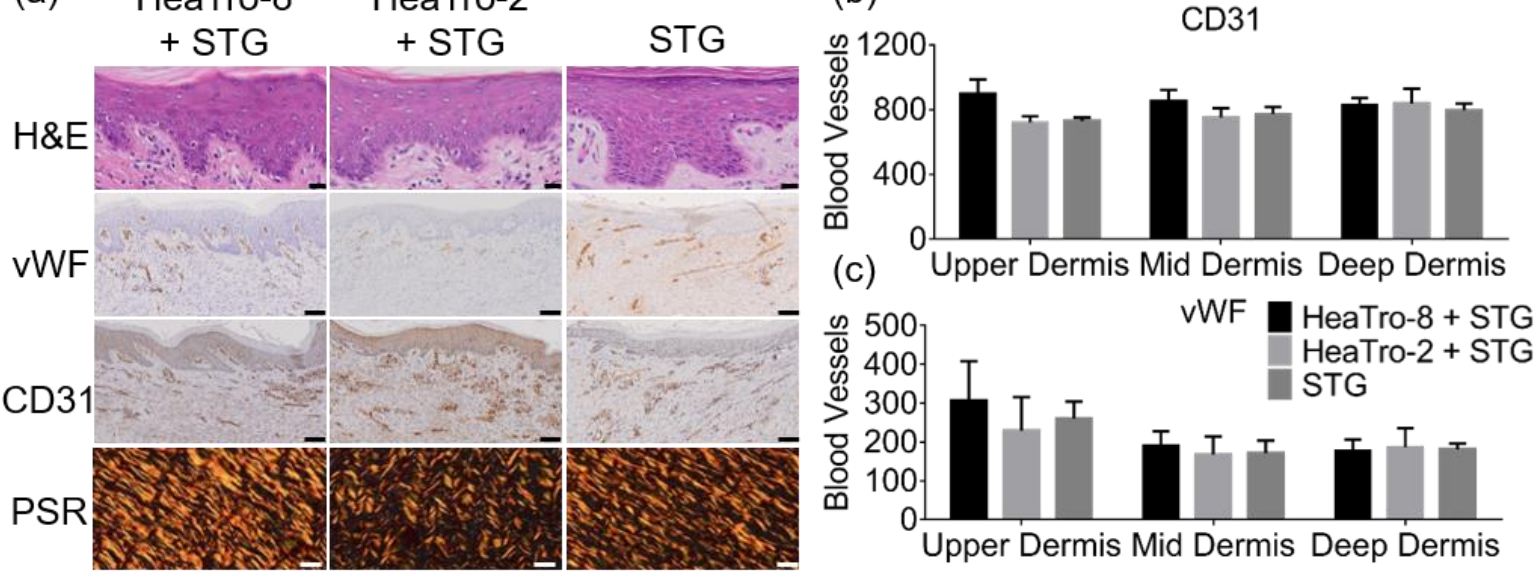

(c) Upper Dermis Mid Dermis Deep Dermis

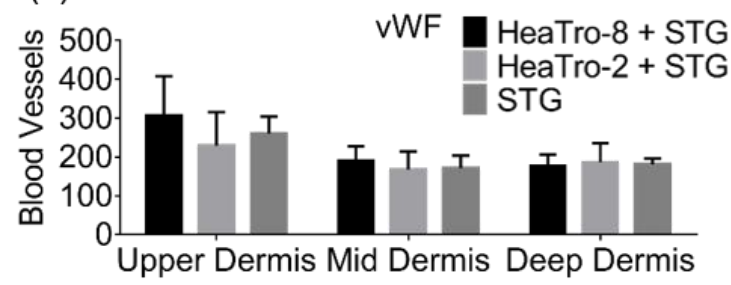

Figure 7. Histology of split thickness grafted pig wounds 14 days after treatment with

HeaTro-8, HeaTro-2, or STG. (a) Representative images of porcine skin sections stained with $\mathrm{H} \& \mathrm{E}$ (scale bar is $20 \mu \mathrm{m}$ ), anti-vWF antibody, anti-CD31 antibody (scale bar is $100 \mu \mathrm{m}$ ),

polarized light PSR (scale bar is $20 \mu \mathrm{m}$ ). Vascularization of grafted wounds in the upper, mid and deep-dermis. Blood vessel (10-100 $\mu \mathrm{m}$ diameter) numbers calculated using (b) CD31, (c) vWF staining. Data presented as mean \pm SEM, $n=3$. Using two-way ANOVA with a Tukey's multiple comparison test no significant differences were found. 


\section{WILEY-VCH}

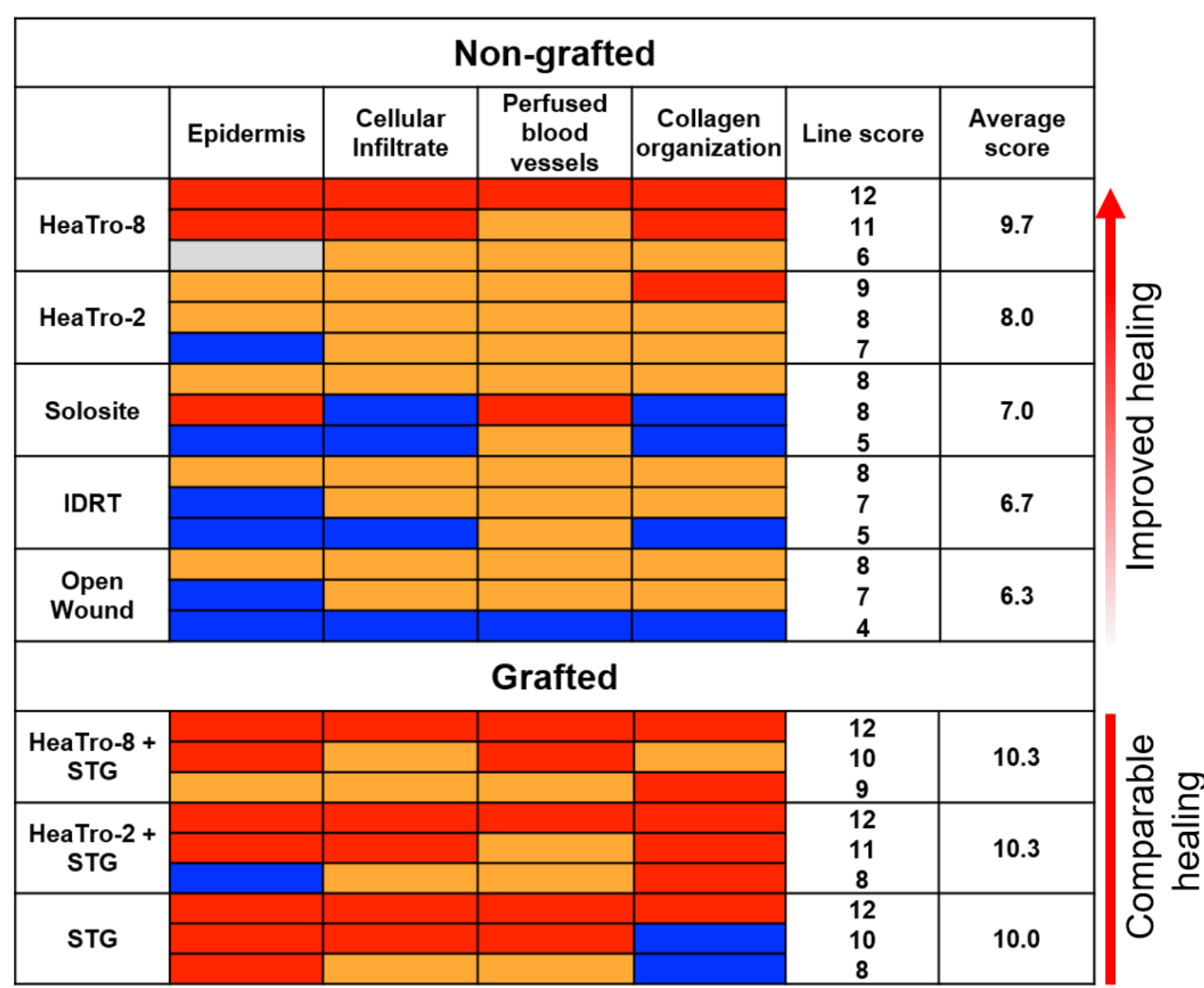

Figure 8. Heat map depicting healing scores for porcine wounds. ${ }^{[35]}$. Blue represents the least advanced (1), orange is intermediate (2), red is best (3). Gray represents the absence of a score due to epidermis removal during histology processing. 


\section{WILEY-VCH}

Table of Contents

Heat-stabilized tropoelastin provides a novel scalable approach to production of elastic

materials. This is attractive because no chemicals are used to generate these elastic structures. The resulting heat-treated tropoelastin promotes the repair of full-thickness wounds.

Keyword: Tropoelastin

S. M. Mithieux, B. Aghaei-Ghareh-Bolagh, L. Yan, K. V. Kuppan, Y. Wang, F. GarcesSuarez, Z. Li, P. K. Maitz, E. Carter, C. Limantoro, W. Chrzanowski, D. Cookson, A. Riboldi-Tunnicliffe, C. Baldock, K. Ohgo, K. K. Kumashiro, G. Edwards, A. S. Weiss*

Tropoelastin implants that accelerate wound repair

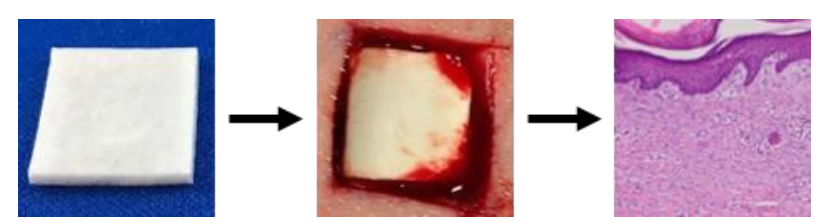




\section{WILEY-VCH}

Copyright WILEY-VCH Verlag GmbH \& Co. KGaA, 69469 Weinheim, Germany, 2016.

Supporting Information

\section{Tropoelastin implants that accelerate wound repair}

Suzanne M. Mithieux, Behnaz Aghaei-Ghareh-Bolagh, Leping Yan, Kekini V. Kuppan, Yiwei Wang, Francia Garces-Suarez, Zhe Li, Peter K. Maitz, Elizabeth Carter, Christina Limantoro, Wojciech Chrzanowski, David Cookson, Alan Riboldi-Tunnicliffe, Clair Baldock, Kosuke Ohgo, Kristin K. Kumashiro, Glenn Edwards and Anthony S. Weiss* 
16 Table S1. Relative intensities of peptide peaks derived from dimer HeaTro and monomer tropoelastin following MALDI-TOF mass spectrometry.

\begin{tabular}{|c|c|c|c|c|c|c|c|c|c|}
\hline \multirow[t]{3}{*}{ Sequence } & \multirow[t]{3}{*}{ Mass } & \multirow[t]{3}{*}{ AA } & \multirow[t]{3}{*}{ MC } & \multirow[t]{3}{*}{ Domain } & \multicolumn{5}{|c|}{ Average Ratio Peak Intensity Monomer/Dimer* } \\
\hline & & & & & \multirow[t]{2}{*}{ Raw Data } & \multicolumn{4}{|c|}{ Data Normalized to AA } \\
\hline & & & & & & $153-183$ & $240-286$ & $442-503$ & $610-647$ \\
\hline GGVPGAIPGGVPGGVFYPGAGLGALGGGALGPGGK & 2888.5 & $1-35$ & 0 & $2-4$ & 2.0 & 1.7 & 1.6 & 1.7 & 1.3 \\
\hline $\begin{array}{l}\text { GGVPGAIPGGVPGGVFYPGAGLGALGGGALGPGGKPLKPVPGGLAGAGLGA } \\
\text { GLGAFPAVTFPGALVPGGVADAAAAYK }\end{array}$ & 6713.6 & $1-78$ & 2 & $2-6$ & 4.0 & 3.3 & 3.2 & 3.4 & 2.5 \\
\hline PLKPVPGGLAGAGLGAGLGAFPAVTFPGALVPGGVADAAA AYK & 3844.1 & $36-78$ & 1 & $4-6$ & 1.2 & 1.1 & 1.1 & 1.0 & 0.7 \\
\hline PVPGGLAGAGLGAGLGAFPAVTFPGALVPGGVADAAAAYK & 3505.9 & $39-78$ & 0 & 4-6 & 3.3 & 3.0 & 2.9 & 2.9 & 2.1 \\
\hline AGAGLGGVPGVGGLGVSAGAVVPQPGAGVK & 2456.4 & $82-111$ & 0 & $6-8$ & 1.6 & 1.4 & 1.3 & 1.4 & 1.0 \\
\hline AGAGLGGVPGVGGLGVSAGAVVPQPGAGVKPGK & 2738.5 & $82-114$ & 1 & $6-8$ & 1.3 & 1.1 & 1.0 & 1.1 & 0.8 \\
\hline VPGVGLPGVYPGGVLPGARFPGVGVLPGVPTGAGVK & 3294.9 & $115-150$ & 0 & $8-10$ & 0.7 & 0.7 & 0.7 & 0.7 & 0.5 \\
\hline VPGVGLPGVYPGGVLPGARFPGVGVLPGVPTGAGVKPK & 3520.0 & $115-152$ & 1 & $8-10$ & 1.9 & 1.6 & 1.6 & 1.6 & 1.2 \\
\hline APGVGGAFAGIPGVGPFGGPQPGVPLGYPIK & 2831.5 & $153-183$ & 0 & $10-12$ & 1.2 & 1.0 & 1.0 & 1.1 & 0.8 \\
\hline LPGGYGLPYTTGK & 1323.7 & $187-199$ & 0 & $12-13$ & 0.8 & 0.6 & 0.6 & 0.6 & 0.5 \\
\hline LPYGYGPGGVAGAAGK & 1434.7 & $200-215$ & 0 & 13-14 & 0.8 & 0.6 & 0.6 & 0.6 & 0.5 \\
\hline AGYPTGTGVGPQAAAAAAAK & 1729.9 & $216-235$ & 0 & 14-15 & 0.8 & 0.7 & 0.7 & 0.7 & 0.5 \\
\hline FGAGAAGVLPGVGGAGVPGVPGAIPGIGGIAGVGTPAAAAAAAAAAK & 3763.1 & $240-286$ & 0 & $15-17$ & 1.3 & 1.0 & 1.0 & 1.1 & 0.8 \\
\hline $\begin{array}{l}\text { YGAAAGLVPGGPGFGPGVVGVPGAGVPGVGVPGAGIPVVPGAGIPGAAVPGVV } \\
\text { SPEAAAK }\end{array}$ & 5076.8 & $290-349$ & 0 & 17-19 & 1.5 & 1.2 & 1.1 & 1.3 & 0.9 \\
\hline $\begin{array}{l}\text { YGARPGVGVGGIPTYGVGAGGFPGFGVGVGGIPGVAGVPSVGGVPGVGGVPG } \\
\text { VGISPEAQAAAAAK }\end{array}$ & 5711.0 & $357-422$ & 0 & $19-21$ & 1.5 & 1.3 & 1.2 & 1.3 & 0.9 \\
\hline YGVGTPAAAAAK & 1076.6 & $426-437$ & 0 & $21-23$ & 0.8 & 0.6 & 0.6 & 0.7 & 0.5 \\
\hline $\begin{array}{l}\text { AAQFGLVPGVGVAPGVGVAPGVGVAPGVGLAPGVGVAPGVGVAPGVGVAPGIG } \\
\text { PGGVAAAAK }\end{array}$ & 5127.9 & $442-503$ & 0 & $23-25$ & 1.2 & 1.0 & 0.9 & 1.0 & 0.7 \\
\hline $\begin{array}{l}\text { AQLRAAAGLGAGIPGLGVGVGVPGLGVGAGVPGLGVGAGVPGFGAVPGALAAA } \\
\text { K }\end{array}$ & 4526.6 & $512-565$ & 0 & $25-27$ & 1.9 & 1.6 & 1.6 & 1.6 & 1.2 \\
\hline YGAAVPGVLGGLGALGGVGIPGGVVGAGPAAAAAAAK & 3014.7 & $569-605$ & 0 & $27-29$ & 1.3 & 1.1 & 1.1 & 1.1 & 0.8 \\
\hline AAQFGLVGAAGLGGLGVGGLGVPGVGGLGGIPPAAAAK & 3154.8 & $610-647$ & 0 & $29-31$ & 1.6 & 1.3 & 1.3 & 1.4 & 1.0 \\
\hline $\begin{array}{l}\text { YGAAGLGGVLGGAGQFPLGGVAARPGFGLSPIFPGGACLGKACGRK\# } \\
\end{array}$ & 4230.2 & 651-696 & 1 & $31-36$ & 2.5 & 2.0 & 2.0 & 2.4 & 1.6 \\
\hline
\end{tabular}

$62 * n=4$ "Peptide contains a missed cleavage due to disulfide bridge. Present in 2 out of 4 HeaTro samples.

63 Highlighted rows identify species for which the average ratio peak intensity is consistently $>2.0$. 
Figure 1

Click here to access/download

Production Data

Figure 1.tif

Figure 1

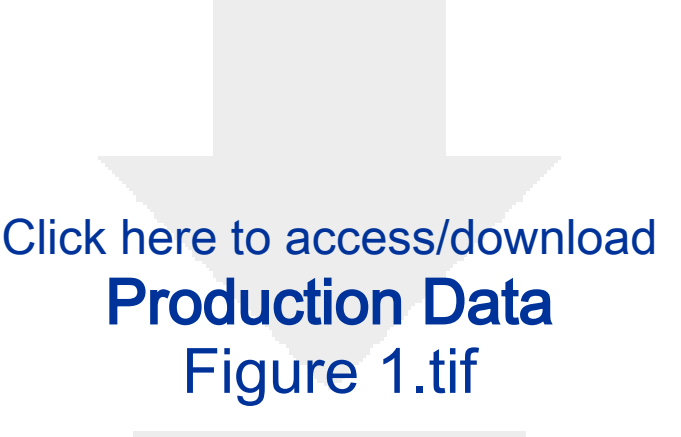


Figure 2

Click here to access/download

Production Data

Figure 2.tif 
Figure 4
\[ \begin{array}{c}\text { Click here to access/download } \\ \text { Production Data } \\ \text { Dirum 1 tif }\end{array} \]

Figure 4 .
\[ \text { Click here to access/download } \]
Production Data
Ficiure 1 tif

Figure 4 .
\[ \text { Click here to access/download } \]
Production Data
Ficiure 1 tif

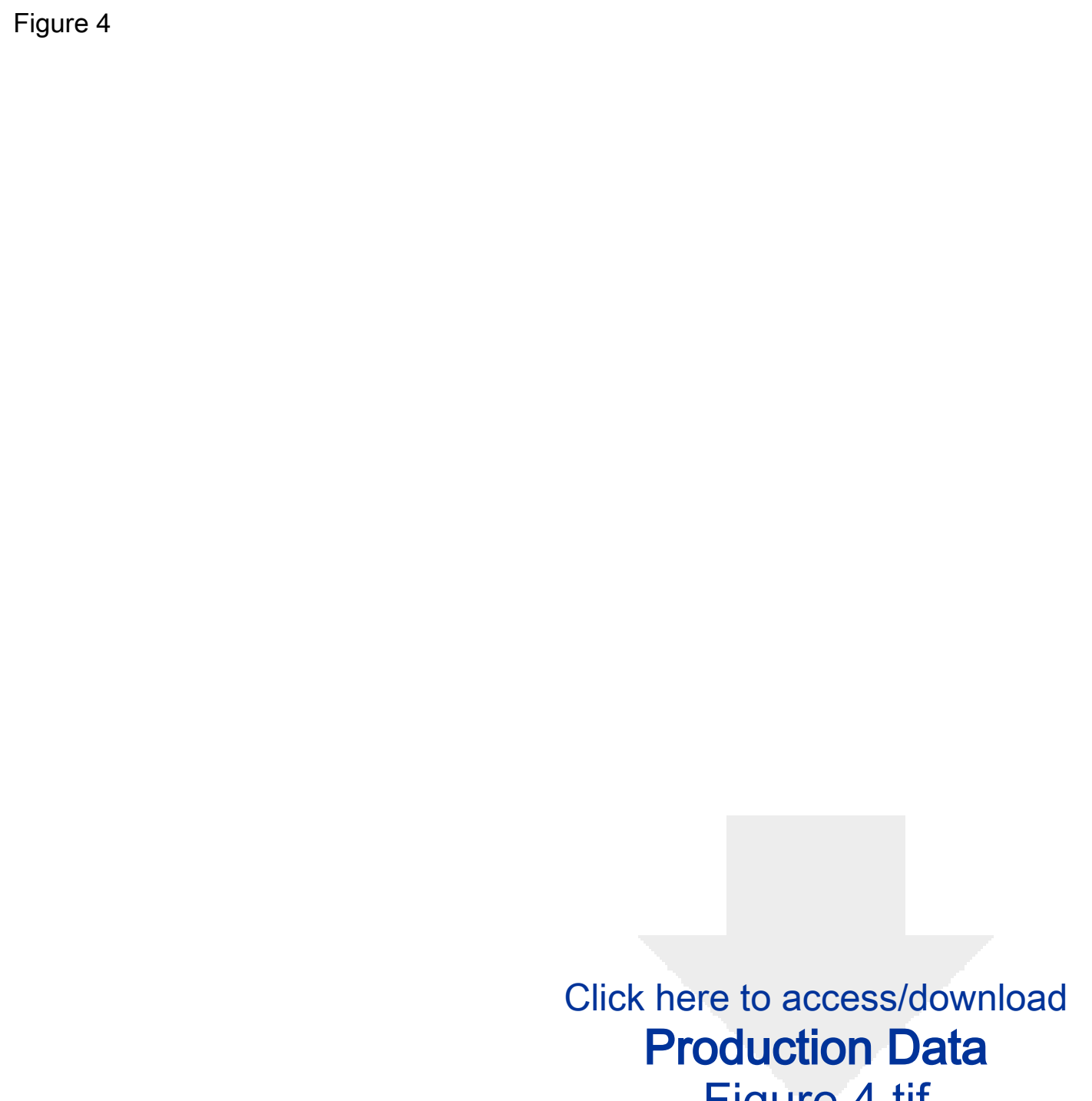

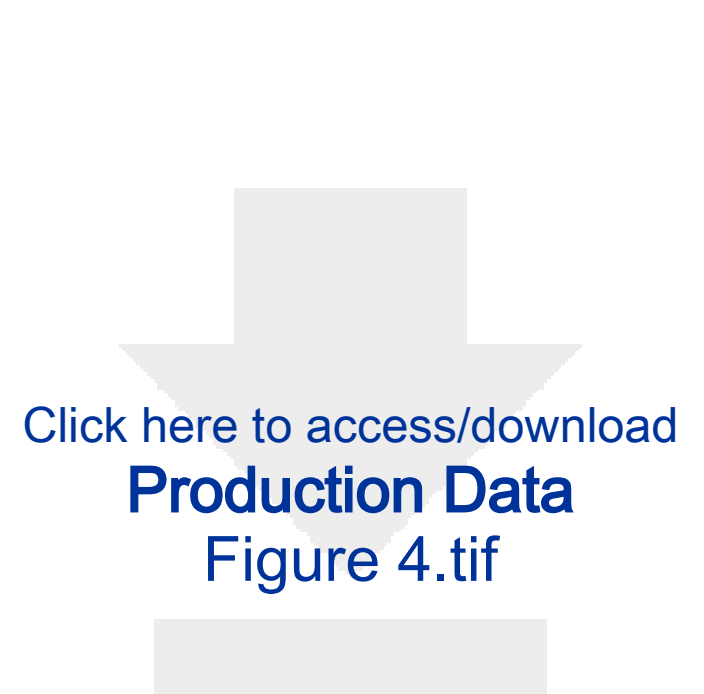

.

\section{Figure 4.tif

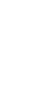

1.

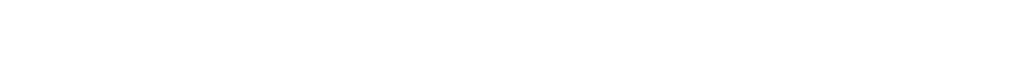

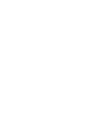

Pron 
Click here to access/download

Figure 5

\section{Production Data
Figure 5.tif \\ Production Data
Figure 5.tif .

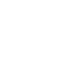

5

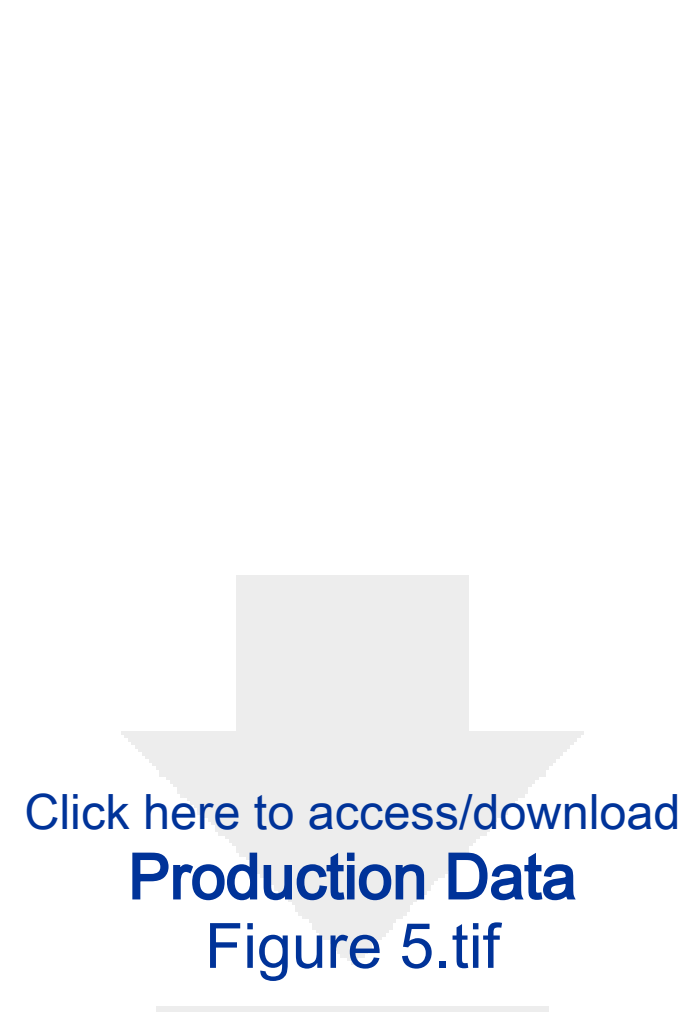

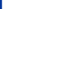

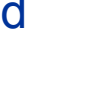

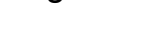

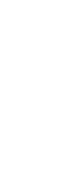

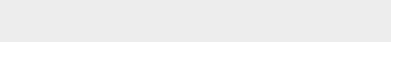

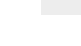

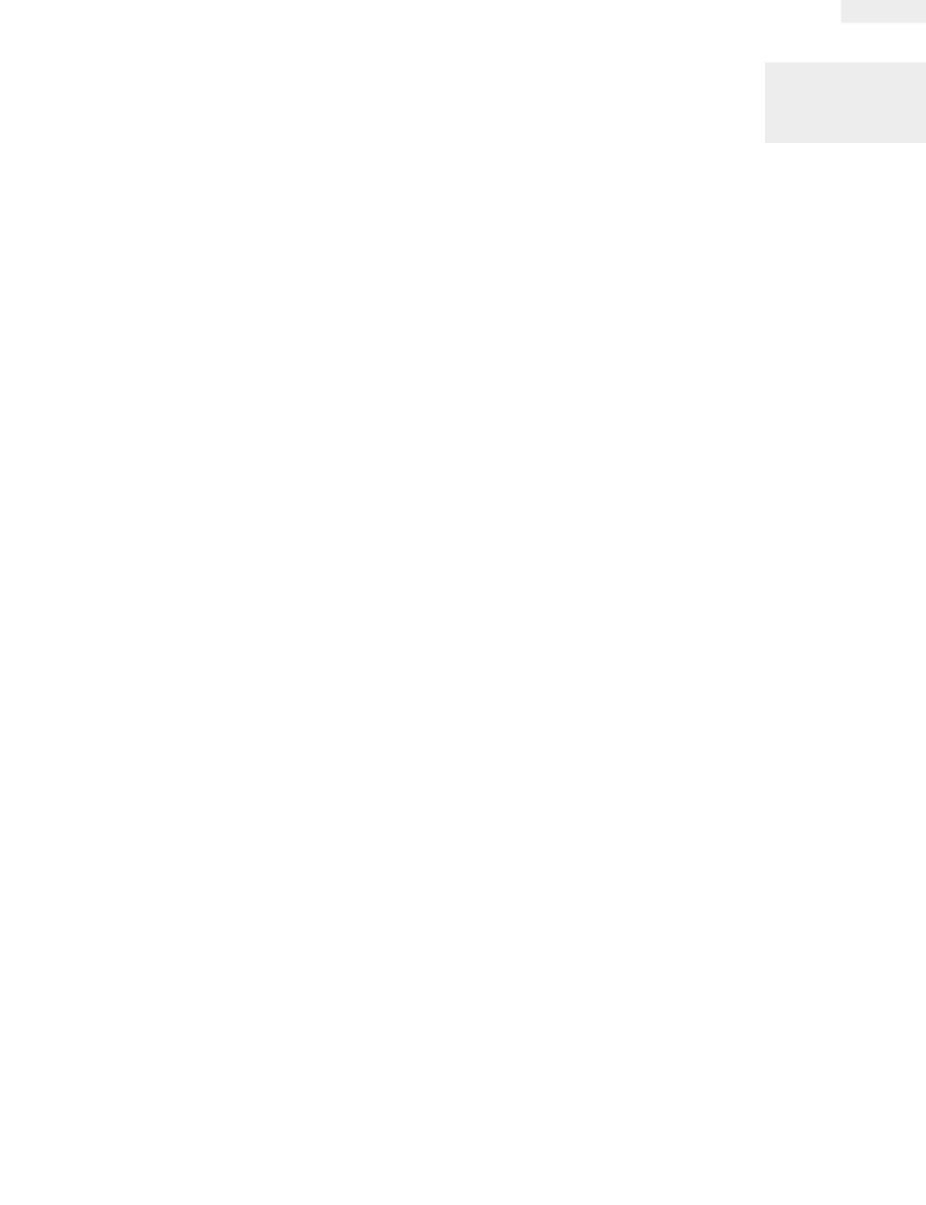

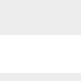


Figure 6

Click here to access/download
Production Data
Figure 6.tif

Click here to access/download
Production Data
Figure 6.tif

Click here to access/download
Production Data
Figure 6.tif

Click here to access/download
Production Data
Figure 6.tif

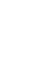


Figure 7

\section{Click here to access/download
Production Data
Figure 7.tif \\ Click here to access/download
Production Data
Figure 7.tif Click here to access/download
Production Data
Figure 7.tif \\ Click here to access/download
Production Data
Figure 7 .tif

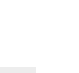


Figure 8

8
Click here to access/download
Production Data
Figure 8.tif Figure 8.tif

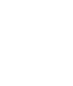


Click here to access/download Production Data ToC.tif 